\title{
Modeling Decomposition of Unconfined Rigid Polyurethane Foam
}

\author{
Michael L. Hobbs, ${ }^{*}$ Kenneth L. Erickson, and Tze Y. Chu \\ Engineering Sciences Center \\ P. O. Box 5800, MS-0834 \\ Sandia National Laboratories ${ }^{\dagger}$ \\ Albuquerque, New Mexico 87185
}

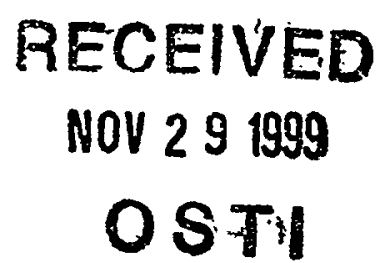

The decomposition of unconfined rigid polyurethane foam has been modeled by a kinetic bond-breaking scheme describing degradation of a primary polymer and formation of a thermally stable secondary polymer. The bond-breaking scheme is resolved using percolation theory to describe evolving polymer fragments. The polymer fragments vaporize according to individual vapor pressures. Kinetic parameters for the model were obtained from Thermal Gravimetric Analysis (TGA). The chemical structure of the foam was determined from the preparation techniques and ingredients used to synthesize the foam. Scale-up effects were investigated by simulating the response of an incident heat flux of $25 \mathrm{~W} / \mathrm{cm}^{2}$ on a partially confined $8.8-\mathrm{cm}$ diameter by $15-\mathrm{cm}$ long right circular cylinder of foam that contained an encapsulated component. Predictions of center, midradial, and component temperatures, as well as regression of the foam surface, were in agreement with measurements using thermocouples and $\mathrm{X}$-ray imaging.

Keywords: polyurethane foam, decomposition, chemical structure, percolation theory, vapor-liquid equilibrium

\section{INTRODUCTION}

Rigid polyurethane foams are used as encapsulants to isolate and support thermally sensitive components within weapon systems. When exposed to abnormal thermal environments, such as fire, various encapsulated components are designed to fail sequentially. In hazards analysis, the thermal response of the encapsulated components depends primarily on the behavior of the foam. Modeling foam decomposition is a difficult problem not only because of the numerical challenges associated with steep reaction fronts but also because of the difficulty of describing important chemical and physical processes, such as non-Newtonian fluid flow. In the current paper, thermal transport and

* Corresponding author, email: mlhobbs@sandia.gov, FAX: (505) 844-8251

$\dagger$ Sandia is a multiprogram laboratory operated by Sandia Corporation, a Lockheed Martin Company, for the United States Department of Energy under Contract DE-AC04-94AL85000. 
decomposition chemistry are discussed in detail. Mass transport, species diffusion, bubble mechanics, fluid flow, and gravitational effects are beyond the scope of this paper.

Simplifying assumptions regarding foam decomposition have been made when predicting the thermal response of encapsulated components. For example, the decomposition of foam has been ignored, by assuming the foam was not present and adjusting surface emissivities to match thermocouple data, or by changing physical properties of the foam at prescribed temperatures. Such approximations were consistent with equivalent approximations necessary for single processor calculations. With the advent of massively parallel computers, high-consequence predictions of foam decomposition can be made with a more fundamental foam decomposition model founded on experimental observations to determine accurate decomposition rates, decomposition species, and physical properties of the evolving solid residue.

Computational models used in hazards analysis at Sandia National Laboratories are designed to accommodate mass loss associated with foam decomposition. For example, Fig. 1 shows a COYOTE ${ }^{1}$ finite element calculation of a block of material containing nonreactive components of various shapes exposed to a constant energy flux. In this calculation, the encapsulating material was assumed to decompose by removing elements from the computational domain based on the element exceeding a specified temperature, without using a realistic decomposition mechanism. Actual tests of polyurethane foam exposed to abnormal thermal environments, such as fire, show the system response to be more complex.

Several groups studying the decomposition of large macromolecules have employed statistical network fragmentation models to describe decomposition chemistry. For example, Solomon and coworkers ${ }^{2}$ have implemented a computationally intensive Monte Carlo technique to describe the breakup of coal. Grant et al. ${ }^{3-5}$ have used pseudo lattice structures referred to as Bethe lattices (or Cayley trees) to obtain closed-form solutions of the network statistics as derived by Fisher and Essam. ${ }^{6}$ These closed form solutions parallel the determination of molecular weight distributions during polymer synthesis leading to the critical condition required to form infinite polymer networks referred to as "gels" by Flory. ${ }^{7}$ In the present paper, Flory's methods of building polymers are used to decompose polymers by assuming that closed rings or cycles cannot form and that bond reactivity is independent of the size of 


\section{DISCLAIMER}

This report was prepared as an account of work sponsored by an agency of the United States Government. Neither the United States Government nor any agency thereof, nor any of their employees, make any warranty, express or implied, or assumes any legal liability or responsibility for the accuracy, completeness, or usefulness of any information, apparatus, product, or process disclosed, or represents that its use would not infringe privately owned rights. Reference herein to any specific commercial product, process, or service by trade name, trademark, manufacturer, or otherwise does not necessarily constitute or imply its endorsement, recommendation, or favoring by the United States Government or any agency thereof. The views and opinions of authors expressed herein do not necessarily state or reflect those of the United States Government or any agency thereof. 


\section{DISCLAIMER}

Portions of this document may be illegible in electronic image products. Images are produced from the best available original document. 
Submitted to Polymer Degradation and Stability

the polymer fragment containing the bond. Bond-breaking is assumed to be a random scission process that describes the extent of reaction.

The PolyUrethane Foam (PUF) decomposition model discussed in the present paper is based on three fundamental aspects of thermal decomposition: 1) a kinetic bond-breaking mechanism, 2) lattice statistics to describe the evolving polymer fragments, and 3) vaporization of the small polymer fragments (oligomers) with high vapor pressures as discussed by Fletcher et al. ${ }^{4-5}$ The Bethe lattice statistical model is referred to as percolation theory, since the technique has been used historically to describe fluid flow through a network of permeable and impermeable sites. To use percolation theory in a bond-breaking mechanism, the intact bonds correspond to impermeable sites and the broken bonds correspond to permeable sites. The PUF model parameters include the Arrhenius kinetics controlling bond-breaking, the initial bridge population, the coordination number, and the average site molecular weight. The initial bridge population is assumed to be composed of both strong bridges and weak bridges. The coordination number describes the connectivity of thermally stable sites by bridges.

In the current paper, the term "polymer" will be used to describe the infinite lattice structure, the term "polymer fragments" refers to the finite fragments resulting from polymer decomposition, and the term "oligomer" will be used to describe the finite fragments that evolve into the gas phase. The term "bridge" is used to represent the part of the polymer structure separating thermally stable "sites." "Bridges" are connected to "sites" by chemical bonds. When a bond is broken, a bridge is also broken. A more detailed discussion of "bridges," "sites," and "bonds" can be found in Section 2.

The remainder of this paper is organized as follows: The next section describes the chemical structure of the polyurethane foam used in this study. Section 3 describes the chemical mechanism used to describe bond-breaking. Section 4 presents a brief description of the lattice statistics using percolation theory as applied to bond-breaking. Section 5 describes the vapor-liquid equilibrium model used to determine the split between liquid formation and gas formation. Section 6 discusses the method used to determine the kinetic coefficients for the bond-breaking reactions and includes a comparison between predicted and measured mass loss for various TGA experiments. Section 7 presents an uncertainty and sensitivity analysis of the PUF model. Section 8 shows a comparison between the predicted shape of a regression front and measured shape of the regression front using X-ray tomography. Some general remarks close the paper. 


\section{CHEMICAL STRUCTURE}

Detailed information regarding the chemical structure of the polyurethane foam is required to predict foam decomposition using lattice statistics. The structural units and resulting polymeric network of many synthesized macromolecules, such as polyurethane foam, can be inferred from the starting materials and the synthesis of the macromolecule. Confirmation of the structure is often obtained using IR, solid-state $\mathrm{NMR}^{8}$ and other analytical chemistry techniques.

The most common chemical structural units of the rigid polyurethane foam and the distribution of these structural units are shown in Fig. 2 and were estimated using proprietary synthesis details by assuming equal reactivity of the hydroxyl groups. Various graphic representations of specific chemical structural units are also shown in Fig. 2. These structural units have been assembled to give a representative picture of the foam. The model foam shows the polyurethane structure as a large matrix (essentially infinite) of toluene diisocyanate (TDI) groups connected by aliphatic bridges made from trimethylol propane (TMP), adipic acid (AA), diethylene glycol (DEG), and small amounts of phthalic anhydride.

Figure 3 shows the three most common structural units divided into sites and bridges. The coordination number and molecular weights for specific structural components are shown in Fig. 3. This information can be used to determine the average coordination number, as well as the average molecular weight of the sites and bridges as discussed further in Section 6 . In the present paper, the sites are assumed to be primarily composed of TMP units and the DEG units. The bridges are composed of the TDI and AA structures.

As bonds break at elevated temperatures, polymer fragments are generated. The fragments, with low molecular weights and corresponding high vapor pressures, evaporate to form gaseous oligomers as shown at the bottom of Fig. 2. Higher molecular weight fragments, with low vapor pressures, remain in the condensed phase.

\section{KINETIC MECHANISM}

In this study, the initial polymer structure begins to degrade between 250 and $350^{\circ} \mathrm{C}$ as bridges between sites are either broken or become thermally stable via the evolution of light gases, similar to the cellulose decomposition model discussed by Grant et al. ${ }^{3}$ and Shafizadeh. ${ }^{9}$ At these temperatures, secondary reactions between functional 
groups can also form bridges between sites, creating a secondary polymer structure. The secondary polymer is assumed to decompose analogously to the primary polymer, but at temperatures above $350^{\circ} \mathrm{C}$ and at a different rate.

The PUF bond decomposition model is an extension of the Grant et al. ${ }^{3}$ bond-breaking scheme and is the first attempt to describe degradation of a polyurethane foam using percolation theory with vapor liquid equilibrium. All of the PUF parameters were obtained from data taken near ambient pressures; and this model is not expected to perform well under heavy confinement where secondary reactions between the vapor and solid are significant. Currently, an experimental program ${ }^{10}$ is being conducted to obtain additional information regarding the decomposition mechanism with attention focused on confinement issues and pressure effects.

The kinetic scheme along with a graphical description is shown in Figs. 4 and 5. This 9 step mechanism with 11 "species" describes four events: 1) competition between local crosslinking and side-chain formation within the primary polymer, 2) competition between side-chain evolution from the primary polymer and the formation of a secondary polymer, 3) competition between local crosslinking in the secondary polymer and side-chain formation within the secondary polymer, and 4) side-chain evolution from the secondary polymer.

The $L, L, C_{1}$, and $C_{2}$ represent bridges as shown in Figs. 4 and 5. A representative labile bridge in the primary polymer, $L$, is the TDI structural component as shown boxed in Fig. 4 . The $\mathcal{L}$ bridge is shown in Fig. 4 connecting two trimethylol propane sites with urethane linkages. The labile bridge in the secondary polymer, $L$, is also shown boxed in Figs. 4 and 5. The $L$ bridge is composed of two toluene components linked by a carbodiimide group, $-\mathrm{N}=\mathrm{C}=\mathrm{N}$ - . The $\mathrm{L}$ bridge is connected to the polymer by two urethane linkages. Thermally stable bridges that connect sites, $c_{1}$ and $c_{2}$, are shown in Figs. 4 and 5 . The thermally stable bridges are actually bonds and do not contain mass. Under certain conditions, decomposing polyurethane foam produces a char, or carbonaceous residue, that is thermally stable. In the present paper, the formation of thermally stable bonds contributes to char formation.

The $\delta, d$, and $g_{i}$ represent side-chains or "danglers" in the primary polymer, side-chains or "danglers" in the secondary polymer, and various gas species, respectively. Representative "danglers" are shown boxed in Figs. 4 and 5. Reversible reactions are included to allow the "danglers" to reattach to the polymer. Such reactions will likely be more significant when the foam is degraded under confinement. This nomenclature closely follows the Grant et al. $^{3-5}$ nomenclature. 
Submitted to Polymer Degradation and Stability

The reaction sequence begins when a weak bond, e.g. the ether bond of the carbomate group, is broken to form either a toluene diisocyanate (TDI) side-chain ( $\delta$ in Fig. 5 ) or evolves as a gas ( $g_{1}$ in Fig. 4 ) with concurrent stabilization of adjacent clusters forming a stable bridge ( $c_{1}$ in Fig. 4). The TDI side-chains, referred to as danglers, may eventually evolve as light gas fragments ( $g_{2}$ in Fig. 4) through subsequent, slower reactions. A secondary polymer may evolve as two TDI side-chains react to form a weak bridge $(\mathrm{L})$ with the subsequent evolution of $\mathrm{CO}_{2}$ denoted as $g_{3}$. The molecular weight of $L$ is twice the molecular weight of $\mathcal{L}$ minus the molecular weight of $g_{3}$. The degradation of the secondary polymer is assumed to be similar to the degradation of the primary polymer except the labile bridge in the secondary polymer has a higher molecular weight.

The proposed mechanism in Figs. 4 and 5 is consistent with preliminary experimental observation. For example, Fig. 6.A shows two bond types that likely break during thermal decomposition: (1) the ester bond of the carbomate group and (2) the ether bond of the ester group. Experimental evidence of bond type (1) breaking is shown in Fig. 6.B where an IR spectra of decomposition gases obtained using isothermal TGA-FTIR analysis shows an increase in the $-\mathrm{N}=\mathrm{C}=\mathrm{O}$ stretch region of the IR spectra (wave number of 2250) from evolving gases sampled at $350^{\circ} \mathrm{C}, 400^{\circ} \mathrm{C}$, and $450^{\circ} \mathrm{C}$. Furthermore, solid-state NMR results, shown in Fig. 6.C, show the depletion of the carbonyls associated with the adipic acid and isocyanate structures. The NMR results are consistent with the bonds labeled (1) and (2) breaking. Although additional information regarding decomposition chemistry is evident in the NMR data, complete analysis was not available. The mechanism in Fig. 5 is also consistent with measured decomposition products such as TDI and trimethoxypropane as determined from desorption tube analysis. ${ }^{10}$

Table 1 gives the mechanism, rate equations, and initial conditions for the PUF model. The general reaction rate, $r_{j}$, for the PUF model bond-breaking scheme is described by:

$$
r_{j}=k_{j}(T) \prod_{i=1}^{11} N_{i}^{\mu_{i j}}, \quad j=1, \ldots 9
$$

where $N_{i}$ represents either population parameters $\mathcal{L}, L, \delta, d, c_{1}, c_{2}$ or species parameters $g_{1}, g_{2}, g_{3}, g_{4}, g_{5}$. The concentration matrix is represented by $\mu_{i j}$, which is given in the footnote of Table 1 . The expressions for the kinetic coefficients, $k_{j}(T)$, are given in an Arrhenius form: 


$$
k_{j}(T)=A_{j} \exp \left(-E_{j} / R T\right)
$$

where $A_{j}(1 / \mathrm{s}), E_{j}(\mathrm{cal} / \mathrm{mol}$ or $\mathrm{J} / \mathrm{mol})$, and $R(1.987 \mathrm{cal} / \mathrm{mol}-\mathrm{K}$ or $8.314 \mathrm{~J} / \mathrm{mol}-\mathrm{K})$ are the pre exponential factors, activation energies, and the universal gas constant, respectively. The species rate of change is given by:

$$
d N_{i} / d t=\sum_{j=1}^{J} v_{i j} r_{j}, \quad i=1, \ldots I,
$$

where $v_{i j}$ are the stoichiometric coefficients of the $j$ th reaction as given in the footnote of Table 1 . The kinetic mechanism requires the Arrhenius parameters, $A_{j}$ and $E_{j}$, to be supplied for each reaction. The initial labile bridge population $\left(\mathcal{L}_{0}\right)$ and the initial stable bridge population $\left(\mathcal{C}_{0}\right)$ need to be specified as an initial condition. These parameters may be related to the extent of polymer curing. The initial value problem described by Eqs. (1)-(3) is solved using $\mathrm{DEBDF},{ }^{11}$ a variable-order, backward-difference, ordinary differential equation solver package.

\section{STATISTICAL MODEL}

Percolation theory using Bethe lattices is used to characterize the degraded foam structure with regard to the size and concentration of finite fragments or oligomers. The population variables determined from the kinetic mechanism can be used to determine the fraction of sites that are connected by bridges. For example, the fraction of intact bridges, $p$, can be determined as follows:

$$
p=L+L+c_{1}+c_{2}
$$

The' mass fraction of finite polymer fragments produced from the thermally degrading foam depends on the population of intact bridges, $p$, determined from the kinetic mechanism and the coordination number, $\sigma+1$. The lattice statistics are independent of the type of bridges connecting sites provided the bridges are distinguished as either broken or intact. Thus, percolation theory can be used with any kinetic mechanism provided that the population of broken bridges $(1-P)$ or intact bridges $(P)$ is determined. ${ }^{3}$ As the number of broken bridges increases, the fraction of finite polymer fragments increases relative to the fraction of sites belonging to the infinite network. The infinite network no longer exists below a critical bridge population of $1 / \sigma$ as shown by Fischer and Essam. ${ }^{6}$ A detailed formulation of percolation theory as used in the present paper is given by Hobbs et al. ${ }^{12}$ 
For Bethe lattices, the number of finite polymer fragments can be determined from the coordination number and the bridge population, $p$. The probability, $F_{\mathrm{n}}$, that any given site is a given member of finite polymer fragment of $n$ sites with $s$ bridges is

$$
F_{n}=a_{n} p^{s}(1-p)^{\tau}
$$

where,

$$
\begin{aligned}
& s=n-1 \\
& \tau=n(\sigma-1)+2 .
\end{aligned}
$$

and

$\tau$ is the number of broken bridges on the perimeter of the polymer fragment with $s$-bridges connecting $n$-sites as shown for the trimer in Fig. 7. The number of different ways to form such fragments is represented by $a_{n}:^{3}$

$$
a_{n}=\frac{(\sigma+1) \Gamma(n \sigma+2)}{(n \sigma+1) \Gamma(n) \Gamma[n(\sigma-1)+3]}
$$

where $\Gamma$ represents the standard gamma function. Equation (5) formally states that the probability that any given bridge belongs to an $n$-mer is the probability that the given bridge is intact or occupied $\left(p^{s}\right)$ multiplied by the probability that the nearest neighbor bridges are broken or unoccupied (1-p $)^{\tau}$ with $a_{n}$ accounting for the distinct number of configurations possible for the $n$-mer. Equations (5)-(8) are discussed in more detail in Reference 12.

The mass fraction and molecular weight of each polymer fragment bin can be determined by relating the total mass and mass associated with finite polymer fragments on a site basis. A site is defined as the portion of the polymer that can be isolated by bridges as shown enclosed in dashed lines in Fig. 8 . The total mass per site is:

$$
m_{t}=M_{a}+\frac{M_{b}}{2}\left(1-c_{o}\right)(\sigma+1)
$$

where $M_{a}$ is the molecular weight of the site as represented by a sphere (or mer) as shown in Fig. $8, M_{b} / 2$ is half the molecular weight of the bridges connecting the spheres, and $\left(1-c_{o}\right)$ represents the fraction of bridges which are labile, and $\sigma+1$ is the coordination number.

The mass of gas released can also be expressed on a site basis as

$$
m_{g}=\left[\left(\frac{M_{b}}{2}\right)\left(g_{1}+g_{2}\right)+\left(\frac{2 M_{b}-M_{g 3}}{2}\right)\left(g_{4}+g_{5}\right)+\left(\frac{M_{g 3}}{2}\right)\left(g_{3}\right)\right](\sigma+1)
$$


Submitted to Polymer Degradation and Stability

where $M_{b}$ is the molecular weight of $g_{1}$ and $g_{2}, 2 M_{b}-M_{g 3}$ is the molecular weight of $g_{4}$ and $g_{5}$, and $M_{g 3}$ is the molecular weight of $g_{3}$. These molecular weights are divided by two to normalize the gas populations to the total mass per site given in Eq. (9) by noting

$$
g_{1}+g_{2}+g_{3}+2 g_{4}+2 g_{5} \rightarrow\left(1-c_{o}\right) \quad \text { as } \quad \text { time } \rightarrow \infty \text {. }
$$

The mass of a finite polymer fragment expressed on a site basis, $m_{n}$, can be determined from the mass of the finite fragment, $M_{n}$, multiplied by the $n$-site polymer fragment population on a site basis, $Q_{n}$ :

$$
\begin{gathered}
m_{n}=M_{n} Q_{n} \\
\text { where, } M_{n}=n M_{a}+(n-1) M_{b}\left(\frac{\mathcal{L}}{p}\right)+(n-1)\left(2 M_{b}-M_{g 3}^{r}\right)\left(\frac{\mathrm{L}}{p}\right)+ \\
\frac{M_{b}}{2}\left[\frac{\tau \delta}{(1-p)}\right]+\frac{\left(2 M_{b}-M_{g 3}\right)}{2}\left[\frac{\tau \mathrm{d}}{(1-p)}\right] \\
\text { and } Q_{n}=F_{n} / n=\left[a_{n} p^{n-1}(1-p)^{n(\sigma-1)+2}\right] / n
\end{gathered}
$$

$M_{n}$ is the molecular weight of the $n^{\text {th }}$-polymer fragment bin. The first term in Eq. (12) represents the number of mers in the n-mer multiplied by the mer molecular weight. The second term in Eq. (12) represents the number of bridges, $n-1$, in the polymer fragment multiplied by the mass of the bridges of type $\mathcal{L}$. The third term in Eq. (12) represents the number of bridges in the polymer fragment multiplied by the mass of the bridges of type $L$. The last two terms in Eq. (12) represent the weight of the side-chains, or "danglers," which can evolve over time. The factors $\delta /(1-p)$ and $d /(1-p)$ represent the fraction of side-chains of type $\delta$ and $d$, respectively. The fraction of broken bridges with one side-chain being formed from each broken bridge is represented by $(1-p)$, and $\tau$ is the number of bridges that isolate a polymer fragment as given in Eq. (6). The factor " 2 " in the denominator of the last two terms in Eq. (12) is needed to be consistent with Eqs. (9) and (10).

The primary variables of interest for the network statistical model are molecular weight of the various gases ( $M_{b}$ is the molecular weight of $\mathrm{g}_{1}$ and $\mathrm{g}_{2}, 2 M_{b}-M_{g 3}$ is the molecular weight of $\mathrm{g}_{4}$ and $\mathrm{g}_{5}$, and $M_{g 3}$ is the molecular weight of $\left.g_{3}\right)$, the mass fraction of the gas $\left(f_{g}=m_{g} / m_{t}\right.$, where $m_{g}$ is calculated from Eq. 10 and $m_{t}$ is calculated from Eq. 9), the molecular weight of the $n^{t h}$-polymer fragment (Eq. 12) and the mass fraction of the $n^{t h}$-polymer fragment 
$\left(f_{n}=m_{n} / m_{t}\right.$, where $m_{n}$ is calculated from Eq. 12 and $m_{t}$ is calculated from Eq. 9). A separate model must be used to determine the amount of finite polymer fragments transported to the gas-phase.

\section{VAPOR-LIQUID EQUILIBRIUM MODEL}

The fraction of the gaseous oligomers can be determined by using a simple vapor-liquid equilibrium relationship using a combination of Dalton's law and Raoult's law. In the current paper, a standard multicomponent isothermal flash calculation was used to determine the split between vapor and condensed phases following the procedure used by Fletcher et al. ${ }^{5}$ Details about the vapor-liquid model can be found in Ref. 12.

A standard multicomponent isothermal flash calculation ${ }^{14}$ is used with the Rachford-Rice equation to determine the split between vapor and liquid by solving the following equation for $V / F$ :

$$
0=\sum_{i=1}^{n} \frac{z_{i}\left(K_{i}-1\right)}{\left(K_{i}-1\right) \frac{V}{F}+1}
$$

where

$$
K_{i}=\frac{y_{i}}{x_{i}}=\frac{P^{*}}{P}
$$

$V I F$ is determined iteratively using the zeroin solver. ${ }^{15}$ The parameters $z_{i}, x_{i}$, and $y_{i}$ represent mole fractions in the feed, liquid phase, and vapor phase, respectively. $V$ and $F$ represent the total moles in the vapor phase and in the feed, respectively. $P^{*}$ represents the vapor pressure of the pure component at the system pressure, $P$. The mole fractions in the liquid and vapor phases can be determined as follows:

$$
\begin{gathered}
x_{i}=\frac{z_{i}}{\left(K_{i}-1\right) \frac{V}{F}+1} \\
\text { and } y_{i}=K_{i} x_{i}=\frac{K_{i} z_{i}}{\left(K_{i}-1\right) \frac{V}{F}+1}
\end{gathered}
$$

The gases $g_{1}$ through $g_{5}$ must be combined with the finite fragments to calculate the feed mole fractions, $z_{i}$. The "K-values" defined in Eq. (16) can be determined from the vapor pressure of the pure components, $P^{*}$, divided 


\section{Submitted to Polymer Degradation and Stability}

by the system pressure, $P$. Fletcher et al. ${ }^{5}$ have proposed the following vapor pressure correlation for high molecular weight organic molecules:

$$
P_{n}^{*}=87100 \exp \left(\frac{-299 M_{n}^{0.590}}{T}\right)
$$

where $P_{n}{ }^{*}$ is the vapor pressure of the pure $n^{t h}$-polymer fragment in atmospheres, $M_{n}$ is the molecular weight of the $n^{t h}$-polymer fragment in $\mathrm{g} / \mathrm{mol}$ as determined from Eq. (12), and $T$ is the temperature in $\mathrm{K}$. The coefficients in Eq. (19) were obtained from coal tars with molecular weights ranging from 110 to $315 \mathrm{~g} / \mathrm{mol}$. The vapor pressure predicted with Eq. (19) increases with lower molecular weight species. The functional form of Eq. (19) is similar to the Clausius-Clapeyron equation.

Fletcher et al. ${ }^{5}$ have shown acceptable agreement with boiling point data for 111 organic compounds at pressures of $0.007,0.08,1$, and 10-atm using the correlation given in Eq. (19). The 111 organic compounds, with molecular weights as high as 244 , did not contain long chain alkanes or compounds with more than two oxygen atoms. Long chain hydrocarbons are not expected to occur in the polyurethane tar in significant quantities. However, some of the expected products measured by Chu et al., ${ }^{10}$ such as trimethoxypropane, contain more than two oxygen atoms.

The most prevalent decomposition products are TDI (see $\mathrm{g}_{1}$ and $\mathrm{g}_{2}$ in Fig. 4) and carbon dioxide $\left(\mathrm{CO}_{2}\right)$. In the current work, experimentally determined vapor pressures ${ }^{16}$ are used for TDI and $\mathrm{CO}_{2}$. Specifically, the TDI vapor pressure was used for $\mathrm{g}_{1}$ and $\mathrm{g}_{2}$; the $\mathrm{CO}_{2}$ vapor pressure was used for $\mathrm{g}_{3}$; and the correlation given in Eq. (19) was used for $g_{4}, g_{5}$, and the polymer fragments determined from percolation theory.

\section{ESTIMATION OF THE PUF DECOMPOSITION MODEL PARAMETERS}

Nominal values of the parameters used in the PUF model are shown in Table 2 with an estimate of the high and low values used in the sensitivity analysis discussed in Section 7.1. The initial bridge population parameters, $p_{o}$ and $c_{o}$, were estimated based on the degree of polymer curing. The coordination number and molecular weights were calculated from the three most common structural units of the foam as shown in Figs. 2 and 3. Figure 3 shows sites made 
from the trimethylol propane units and the diethylene glycol units. The bridges are composed of the TDI and adipic acid structures. Table 3 shows how the average molecular weights and coordination numbers for the $60 \%, 20 \%$, and $10 \%$ most probable structural units were calculated. The molecular weights and coordination number in Table 2 were determined by taking the probability normalized molecular weights and coordination numbers from Table 3 as follows:

$$
\begin{gathered}
M_{a}=\frac{0.6}{0.9}(131)+\frac{0.2}{0.9}(118)+\frac{0.1}{0.9}(104)=125 \mathrm{~g} / \mathrm{mol} \\
M_{b}=\frac{0.6}{0.9}(163)+\frac{0.2}{0.9}(160)+\frac{0.1}{0.9}(155)=161 \mathrm{~g} / \mathrm{mol} \\
\sigma+1=\frac{0.6}{0.9}(3)+\frac{0.2}{0.9}(2.5)+\frac{0.1}{0.9}(2)=2.8
\end{gathered}
$$

The Arrhenius parameters were obtained by minimizing the root mean squared error between the calculated and measured mass loss for six, unconfined, isothermal TGA experiments using DAKOTA (Design Analysis Kit for OpTimizAtion, Ref. 17). The unconfined TGA experiments utilized nominally 5-mg samples in open platinum pans and were designed to examine decomposition mechanisms under conditions that minimize mass transfer and reversible and secondary reactions. ${ }^{10}$ Figure 9.A and 9.B show a comparison between predicted and measured condensed mass fractions (foam mass measured by the TGA divided by the initial foam mass) for the isothermal TGA experiments used to obtain the kinetic parameters. The single isothermal TGA samples were quickly ramped ( 10-15 minutes) to the temperatures indicated in Fig. 9.A. The dual isothermal TGA samples were ramped to $300^{\circ} \mathrm{C}$ and held for about two hours then ramped to the temperatures indicated in Fig. 9.B. The three single isothermal experiments shown in Fig. 9.A were intended to isolate the kinetics for the decomposition of the initial polymer and formation of the secondary polymer as discussed in more detail by Erickson et al. ${ }^{10}$ The final three isothermal experiments shown in Fig. 9.B were ramped to $300^{\circ} \mathrm{C}$ and held until mass loss was minimal and then ramped to the final isothermal temperature of $380^{\circ} \mathrm{C}, 400^{\circ} \mathrm{C}$, and $420^{\circ} \mathrm{C}$, respectively. The intent of these final three experiments was to isolate the kinetics of the decomposition of the secondary polymer.

The predicted solid fraction is greater than the measured solid fraction for the $270^{\circ} \mathrm{C}$ isothermal TGA results shown in Fig. 9.A. The difference between the predicted and measured solid fraction is not as pronounced at $300^{\circ} \mathrm{C}$ and $330^{\circ} \mathrm{C}$. At the lower temperature, alternate pathways involving evolution of a site, such as a trimethylol 
Submitted to Polymer Degradation and Stability

propane with subsequent crosslinking of the resulting urethane danglers, may explain the difference. Future work will address this issue.

The kinetic parameters, determined from the six isothermal TGA experiments, are given in Table 2. Three nonisothermal experiments, not used to estimate kinetic parameters, were simulated to validate the selection of the kinetic parameters for the PUF model. Fig. 9.C shows good agreement between predicted and measured condensed mass fraction for the three nonisothermal TGA experiments with heating rates of 5,20 , and $50-^{\circ} \mathrm{C} / \mathrm{min}$. Figure 10 shows the predicted solid fraction and measured solid fraction for the $20{ }^{\circ} \mathrm{C} / \mathrm{min}$ TGA case for a $4.687-\mathrm{mg}$ sample and a 13.765 -mg sample. Slight differences in the experimental results are probably related to mass transport effects. However, both sets of data show an initial rapid decomposition followed by a slower rate of solid mass loss, which is in agreement with the predictions.

The PUF model does not consider mass transport limitations or mechanical effects such as thermal expansion. Not including these physical phenomena may be acceptable for certain experimental conditions, such as when small samples are considered in the TGA experiments, or when the heating rates are high as in large-scale experiments discussed in Section 8. Under these conditions, diffusion lengths are small and mass transport limitations are negligible. For conditions that favor thicker decomposition fronts, such as confinement and/or low heating rates, the PUF model should be improved with additional physics.

Figure 11 shows solid mass fraction, population parameters, the gas and oligomer mass fractions, and gas molecular weight for the $5-^{\circ} \mathrm{C} / \mathrm{min}$ ramped TGA experiment. Between 0 and 40 minutes in Fig. 11.B, $P$ is shown to be the sum of $\mathcal{L}$ and $c_{1}$. At 40 minutes, $\delta$ starts to decrease with a corresponding increase in $\mathcal{L}$, a result of side-chain cross-linking of $\delta$ to form the bridge $\mathcal{L}_{.}$At 50 minutes, the secondary bridge, L, begins to form as a result of reaction between two $\delta$ side-chains. The increase in both $L$ and $L$ causes the bridge population, $p$, to increase between 40 and 50 minutes. Decay of the weak bridge, $\mathcal{L}$, is shown as the side-chain population $\delta$ increases. The strong bridge formation in the primary and secondary polymer is minimal. However, the formation of strong bridges may become significant at elevated pressures. 
Submitted to Polymer Degradation and Stability

\section{UNCERTAINTY AND SENSITIVITY ANALYSIS OF THE PUF MODEL}

Two techniques were used to analyze parameters in the decomposition model: 1) a variance analysis discussed in Section 7.1 and 2) a response derivative analysis discussed in Section7.3. A grid refinement analysis is also reported in Section 7.2 to establish that solutions are not dependent on the size of elements used in the simulations.

The variance analysis is used to examine primary PUF model parameters that effect the prediction of condensed mass fraction for the $20-^{\circ} \mathrm{C} / \mathrm{min}$ ramped TGA experiment. Parameters with primary effects were the initial bridge population, $P_{o}$; the lattice coordination number, $\sigma+1$; molecular weight of the bridging structures, $M w_{b}$; and several activation energies associated with the formation of a secondary polymer, $E_{i}$.

Two response derivative analyses are performed for fast $(0.97 \pm 0.14 \mathrm{~cm} / \mathrm{min})$ and slow $(0.26 \pm 0.05 \mathrm{~cm} / \mathrm{min})$ burning conditions. ${ }^{*}$ For the fast burn analysis, the most sensitive parameters are the activation energies associated with the formation of the secondary polymer. For the slow burn analysis, the most sensitive parameters are associated with the thermophysical properties of the foam and the destruction of the secondary polymer.

\subsection{VARIANCE ANALYSIS}

To determine the primary effects of various PUF model parameters, a simple sensitivity analysis is performed on 15 parameters using a two-level 20-run Plackett Burman ${ }^{18}$ analysis. Typically, Plackett Burman techniques are typically used in experimental design to determine variables that have primary effects on response variables. Primary effects are determined by comparing calculated factor effects with probability points of a 2-sided t-distribution with 4 degrees of freedom. One degree of freedom is used to estimate the mean. The primary response variable for this study is chosen to be the condensed mass fraction at various times during decomposition of the foam ramped at $20-^{\circ} \mathrm{C} / \mathrm{min}$ as in the thermogravimetric analysis (TGA). The 15 parameters used for this study are given in Table 2. The sensitivity of $M_{g 3}$, was not determined. The 20-run Plackett Burman design used in this study is given in Ref. 18 .

To determine significant factor effects using Plackett Burman analyses, a low value and high value for each of the PUF variables are selected as shown in Table 2. The high and low values for the activation energies are taken as the nominal value $\pm 10 \%$. The molecular weight values are taken from the $10 \%$ and $60 \%$ most probable structures.

* Burning is defined as material destruction by heat. 
Submitted to Polymer Degradation and Stability

Twenty simulations of the TGA experiment with a $20-^{\circ} \mathrm{C} / \mathrm{min}$. ramp are run with the parameters set to either the low value or the high value as described in Ref. 18. The condensed mass fraction at various times is used as the response variable. From the response at these times, a factor effect is calculated using methods discussed in Ref. 18. The significant factors based on a $90 \%$ confidence interval are shaded and boxed in Table 4 . The factor effects before 480 seconds are zero since the temperature is below significant reaction thresholds.

The Plackett Burman analyses indicate that the three structural parameters, $P_{o}, \sigma+1$, and $M_{b}$, are significant in the early decomposition phase, since the significant factor effects are only present in the early stages of mass loss between 480 and 780 seconds. These results are dependent on the selection of the low and high values of each parameter. The parameters with a small difference between the high and low values, such as pressure, are shown to have small factor effects. If the difference between the high and low values were greater, the factor effect may have been more significant. Specification of the initial bridge population, $P_{o}$, is more significant than specification of the initial strong bridge population, $c_{o}$, as shown in Table 4. The lattice coordination number, $\sigma+1$, and the molecular weight of bridges are also significant factors that describe the chemical structure of the foam.

The Plackett Burman analysis also shows that all of the steps in the bond-breaking scheme are significant except for reaction 7 in Table 1. The analysis also indicates that the most sensitive pathway is the formation of the secondary polymer. At the expense of losing generality, the PUF model could possibly be simplified for the 20- ${ }^{\circ} \mathrm{C} / \mathrm{min}$ TGA simulation.

\subsection{GRID-INDEPENDENT SOLUTIONS USING FINITE ELEMENT ANALYSIS}

Calculation of the object function for the response derivative analysis discussed in Section 7.3 requires grid-independent solutions using a finite element heat conduction code. The PUF decomposition model is implemented into the

finite element heat transfer code, COYOTE, ${ }^{1}$ employing a user subroutine. COYOTE is a two- or three-dimensional, finite element, massively parallel computer program designed for analysis of nonlinear heat conduction problems. In addition to solving standard thermal diffusion problems, COYOTE includes the effect of phase change, condensed-phase chemistry, and enclosure radiation. Material deletion is included through the use of the finite element "death" capability. Elements can be removed to create a "dynamic" radiation enclosure. Viewfactors are recalculated whenever an element dies. Gaps at material interfaces are modeled as contact surfaces. Material properties can 
be temperature- and/or species-dependent and either isotropic or orthotropic. A wide variety of boundary conditions are supported in COYOTE and pre and postprocessing file formats are used which permit integration with existing meshing and graphics visualization programs. For the simulations reported in the current work, elements are dynamically removed from the simulation when the condensed fraction within an element dropped below $1 \%$. The velocity of the decomposition front, referred in this paper as the burn front, is determined from the time of element death.

The one-dimensional simulation of burning foam was performed with COYOTE using a single column of elements as shown on the left side of Fig. 12. Three sides of the column of elements were insulated and the fourth side was exposed to various boundary conditions such as radiation. Experimentally determined ${ }^{19}$ thermal conductivity and specific heat were used in the one-dimensional simulations. Several methods can be used to determine the grid sensitivity of decomposing polyurethane foam. For example, the size of the element can be reduced until there is no change in the burn front velocity as shown in the plot of Fig. 12. Another method would be to specify a sufficient number of elements across the reaction front to resolve steep gradients in temperature or species profiles.

The grid error in the legend of Fig. 12 is defined as

$$
\text { grid error }=100 \times\left(V_{i}-V\right) / V_{i}
$$

where $V$ represents the burn velocity and $V_{i}$ represents the grid-independent burn velocity. The grid-independent burn velocity in Fig. 12 is $1.14 \mathrm{~cm} / \mathrm{min}$. Figure 13 shows the grid error for a one-dimensional model with two separate constant flux boundary conditions. The grid-independent burn velocity, $V_{i}$, is equal to $0.64 \mathrm{~cm} / \mathrm{min}$ and 0.31 $\mathrm{cm} / \mathrm{min}$ for the $1.25 \mathrm{cal} / \mathrm{cm}^{2}$-s and $0.6 \mathrm{cal} / \mathrm{cm}^{2}$-s boundary conditions, respectively.

In Fig. 13, the predicted velocity using 2 -mm grids is $0.54 \mathrm{~cm} / \mathrm{min}$ giving a grid error of $15 \%$ for the 1.25 $\mathrm{cal} / \mathrm{cm}^{2}$-s case. Simulations using elements smaller than $2-\mathrm{mm}$ predict faster burn rates than $0.54 \mathrm{~cm} / \mathrm{min}$. The faster burn rates for the simulations using smaller elements is related to element death. For example, in the simulations using smaller elements, the criteria for element removal, condensed fraction being less than 0.01 , is satisfied earlier than the criteria would be satisfied for a simulation using larger elements. The smaller elements are removed from the computational domain and the boundary condition is then applied to the newly exposed element. For an element size of $0.5-\mathrm{mm}$, the grid error is less than five percent. The temperature gradient across each element is also an indi- 


\section{Submitted to Polymer Degradation and Stability}

cation of when grid independence is achieved. For example, grid independence in the present study was achieved when the temperature gradient across a dying element was approximately $50^{\circ} \mathrm{C}$ or less.

\subsection{RESPONSE DERIVATIVE ANALYSIS}

The object-oriented optimization code, DAKOTA, ${ }^{17}$ used to determine the PUF model activation energies, can also be used to determine the gradient of an object function with respect to model parameters. This response derivative can be used to quantitatively determine the uncertainty in predicted results. In this paper, two response derivative analyses were performed for fast $(0.97 \pm 0.14 \mathrm{~cm} / \mathrm{min})$ and slow $(0.26 \pm 0.05 \mathrm{~cm} / \mathrm{min})$ burning conditions. For the response derivative analysis, the object function was chosen to be the steady-state burn velocity for one-dimensional foam decomposition, as discussed in Section 7.2. The TGA simulations, discussed in Section 7.1, did not require solution of an energy equation, since the temperature of the foam samples was specified. However, the regression of the decomposition front depends on the thermophysical properties of the foam, as well as the decomposition mechanism. Temperature-dependent thermophysical properties for a rigid polyurethane foam are given in Table 5. The temperature-dependent thermal conductivity and the heat capacity were measured ${ }^{19}$ with a differential scanning calorimeter and a laser flash technique, respectively.

Two different rates were investigated: 1$)$ a fast burn rate $(0.97 \pm 0.14 \mathrm{~cm} / \mathrm{min})$ obtained using a radiative boundary condition with a source temperature of $1,000^{\circ} \mathrm{C}$ and 2) a slow burn rate $(0.26 \pm 0.05 \mathrm{~cm} / \mathrm{min})$ obtained using a constant flux boundary condition of $0.5 \mathrm{cal} / \mathrm{cm}^{2}$-s. All the simulations in this section were made using $0.5-\mathrm{mm}$ elements. The gradient of the object function with respect to model parameters, $d V / d \zeta_{i}$, was used to determine the uncertainty in burn velocity with the following equation:

$$
(\Delta V)^{2}=\sum_{i=1}^{n}\left(\Delta \zeta_{i} \frac{d V}{d \zeta_{i}}\right)^{2}
$$

where $V, \zeta_{i}, \Delta V$, and $\Delta \zeta_{i}$ represent the burn velocity, the $i^{\text {th }}$ model parameter, uncertainty in burn velocity, and the uncertainty in the $i^{\text {th }}$ parameter, respectively. The subscript $i$ represents the initial density of the foam, the conductivity of the foam, the heat capacity of the foam, the initial temperature of the foam, the activation energies of the nine PUF reactions, the decomposition enthalpy, and the emissivity of the foam. Since the thermal conductivity and heat 
capacity of the foam are temperature-dependent, the sensitivity parameter for these variables was modified by using a multiplying factor to scale the temperature-dependent property. The scale factor for both the thermal conductivity and specific heat was chosen to be one. A similar approach was used for the reaction enthalpy.

Table 6 gives the 15 parameters used in the response derivative analysis of fast burn rate analysis obtained using the one-dimensional burn model with a radiative boundary condition. The nominal parameter values, $\zeta_{i}$; the gradient of the burn velocity, $d V / d \zeta_{i}$; the estimated parameter uncertainty, $\Delta \zeta_{i}$; and the individual variable uncertainty, $\left(\Delta \zeta_{i} \frac{d V}{d \zeta_{i}}\right)^{2}$; are also given in Table 6. The contributions to the overall uncertainty are shown in parenthesis in Table 6. Variables with a contribution to the overall uncertainty greater than $5 \%$ are shaded. The most sensitive variables affecting burn velocity for the case with the radiative boundary condition are the activation energies associated with the Reactions 2,4 , and 5 as given in Table 1 . These are the same kinetic variables that were determined to be the most sensitive kinetic variables in the Plackett Burman analysis discussed in Section 7.1. A one percent change in model parameters resulted in a $14 \%$ change in the high burn velocity case.

The sensitivity of the PUF model parameters to a moderate boundary condition of $0.5 \mathrm{cal} / \mathrm{cm}^{2}$-s was also investigated. The moderate boundary condition resulted in a thicker reaction zone making variables such as thermal conductivity more significant. As highlighted in Table 7, the most sensitive variables affecting burn velocity for the constant flux calculation are the initial density, thermal conductivity, kinetics affecting the formation and destruction of the secondary polymer, and the reaction enthalpy. A one percent variation in the input factors resulted in an $18 \%$ change in the burn velocity. The sensitivity of the model to input parameters show that the foam decomposition process is highly nonlinear. However, since the burn velocity gradients contain both positive and negative values, the $18 \%$ variation in burn velocity is a conservative estimate.

\section{SCALE-UP EXPERIMENT}

Chu et al. ${ }^{10}$ have performed component-scale experiments of foam decomposition with well-characterized boundary conditions using a heat lamp array to produce fire-like heat fluxes. The two-dimensional axisymmetric geometry of the foam experiment is shown in Fig. 14 with an $8.8-\mathrm{cm}$ diameter by $15-\mathrm{cm}$ long right circular cylinder of foam encapsulating a $3.8-\mathrm{cm}$ diameter by $6.4-\mathrm{cm}$ long right circular cylinder of 304 stainless steel (SS). The face of the 


\section{Submitted to Polymer Degradation and Stability}

embedded component was 3.2-cm from the heated surface. The foam cylinder was contained in a sample cup that is shown in Fig. 15. The cup consisted of a $0.95-\mathrm{cm}$ thick stainless steel bottom, which was press fit into a 7.3-cm long, thin wall $(0.5-\mathrm{mm})$ stainless steel tube. Six $6-\mathrm{mm}$ diameter holes were drilled through the side of the stainless steel tub, near the cup bottom, to vent decomposition gases. The number and size of the vents were chosen to keep the pressure within the cup near ambient conditions to minimize mass transfer effects. Figure 16 shows the vent holes in an X-ray image of the component-scale experiment after 15 minutes exposure to an incident flux of $25 \mathrm{~W} / \mathrm{cm}^{2}$. The burn front is also visible in Fig. 16.

The white circles in Fig. 16 show the locations of the thermocouples that were welded to the side and the bottom of the can. The locations of the thermocouples on the can are shown in more detail in the insert of Fig. 17. Figure 17 also gives the measured temperatures at various axial can locations as well as the temperatures of the bottom of the can for the component-scale experiment. The boundary conditions for the COYOTE simulation of the component-scale experiment were set to the specific temperatures measured along the surface of the confining cup. The cup bottom temperature was maintained at $1,000^{\circ} \mathrm{C}$ with the thermocouple located within the bottom plate as shown in Fig. 17. Figure 18 shows the measured cup wall temperatures and bottom cup temperature at early times. Note in Fig. 18 that temperatures measured with thermocouples $\mathbf{9}$ and 6 should not be hotter than the temperatures measured with thermocouple 4 .

Figure 19 shows the axisymmetric mesh and an X-ray of the foam with the encapsulated 304 SS component. A 3-mm gap was visible in the X-ray taken prior to the experiment. The 3-mm gap was modeled as a radiation enclosure. The element dimensions were chosen to be $5-\mathrm{mm}$ by $5-\mathrm{mm}$ to maintain at least three to four elements across the reaction zone as shown in Fig. 20. The mesh in Fig. 19 is expected to give a grid error, as defined by Eq. (23), less than 5\%. The temperature and density profiles across the decomposition front are shown in Fig. 20.C. The thickness of the front estimated from Fig. 20.C is $4-\mathrm{mm}$, which confirms that the $0.5-\mathrm{mm}$ element dimension is sufficiently small. Figure 20.D also shows the predicted temperature profile and solid fraction profile across the burn front. The density profile, shown in Fig. 20.C, has significant scatter. The density profile was estimated from the pixel density in the X-ray image shown in Fig. 20.A. Despite this uncertainty, the measured density profile and the predicted solid fraction profile across the burn front are similar. 
Figure 21 shows a comparison between X-ray images and calculated temperature profiles of the foam with the cup bottom exposed to an incident flux of $\sim 25 \mathrm{~W} / \mathrm{cm}^{2}$. The boundary conditions were set to the specific temperatures measured along the cup surface and bottom as shown in Fig. 17. The nominal values of the PUF model parameters given in Table 2 and the temperature-dependent thermophysical properties given in Table 5 were used for the calculations shown in Fig. 21. Resistance across the foam residue on the face of the component was also accounted for by assuming a reduced component surface emissivity of 0.6 . The reaction enthalpy was chosen to be consistent with bond energies of polyatomic compounds. In Fig. 21, the decomposition front is horizontal prior to reaching the component. As the front gets closer to the embedded component, the decomposition front curves around the component. The exact location of the decomposition front is seen as a density variation in the X-rays. COYOTE's element death option was used to remove elements when the condensed fraction within individual elements was less than 0.01. The shape of the front is difficult to determine near the wall in the X-rays because of the curvature of the confinement. Nevertheless, the calculated and measured shapes of the decomposition front appear to agree very well.

Figure 22 shows the location of the thermocouples at the center and midradial positions. Figures 23, 24, and 25 show a comparison between predicted and measured center, midradial, and component temperatures, respectively. The encapsulated component appears to heat up the bulk of the foam as shown by the temperature profile labeled (10 in Fig. 23. In previous experiments without an encapsulated component, ${ }^{10}$ all temperature measurements remained essentially constant until the decomposition front was within close proximity. With the encapsulated component, the temperature at the various thermocouple locations is shown to increase gradually until the decomposition front is within close proximity, and the temperature increase is more rapid. This behavior is shown to be more pronounced in the experimental temperature profiles plotted in Figs. 23 and 24. In Fig. 23, the temperature measured by thermocouple (10) appears to be $100{ }^{\circ} \mathrm{C}$ higher than the predicted temperature at that location. This difference between the measurement and calculation is thought to be related to premature exposure of the thermocouple sheath, as shown in Fig. 26 , to the hot radiating can and subsequent heat transfer by conduction to the thermocouple tip. Since the foam burns faster along the wall than in the center of the foam, the thermocouples are likely to become partially exposed. The exposed thermocouples can become hot by radiative energy exchange with the hot boundary. Furthermore, heat transfer between the hot tube walls and the thermocouple leads may have also resulted in unaccounted heat transfer to the thermocouple junctions. 
Submitted to Polymer Degradation and Stability

Figure 27 shows the predicted transmitted heat flux along the center of the foam at various times. The maximum transmitted heat flux within the foam at each time plotted in Fig. 28 was located at the burn front. Furthermore, the overall maximum transmitted heat flux through the foam for all times occurred at early times when the foam surface was intimate with the heated surface. As the burn front moved away from the heated surface, the maximum transmitted heat flux decreased. The decrease in maximum transmitted heat flux is likely due to a view factor effect since gas opacity and convective heat transfer was not included in the model.

Figure 28 shows the predicted center and midradial burn front velocities. The velocities were estimated using the time of element death. The center burn front velocity was slightly greater than the midradial burn velocities, resulting in a curved burn front shown previously in Fig. 21 . The center burn front velocity profile stops at about 7 minutes when the burn front reaches the embedded component.

The 2-D axisymmetric mesh contained 11,209 elements as shown in Fig. 19. The simulation required 11 days and 16 hours of CPU time on a single processor, 3 days 16 hours of CPU time on four processors, and 2 days 1 hour on eight processors. Ninety percent of the CPU time was attributed to the view factor computation while only nine percent of the computation was attributed to the PUF decomposition model. Adaptive gridding would reduce the chemistry computation considerably and increase simulation accuracy, but the view factor CPU time would remain essentially the same on a single-processor machine and could in fact increase if the adaptive grid refinement approaches resolution scales defined in Section 7.2.

\section{SUMMARY, CONCLUSIONS, AND FUTURE WORK}

The PolyUrethane Foam (PUF) decomposition model has been applied to thermal decomposition of rigid polyurethane foam using the three-dimensional finite element code COYOTE. The PUF model is comprised of a kinetic mechanism that describes bond-breaking, a lattice statistics model to describe the mass fraction of finite polymer fragments isolated from the macromolecule by broken bonds, and a transport model to describe the evaporation of the finite polymer fragments with high vapor pressures into the gas phase. The chemical structure of the rigid polyurethane foam was determined from specific synthesis procedures, and the PUF model parameters were obtained from the most probable structural units of the foam. Kinetic parameters for the PUF bond-breaking mechanism were obtained by using an object-oriented optimization code, used to minimize the difference between weight loss mea- 
sured in a small-scale Thermal Gravimetric Analysis (TGA) apparatus using nominally 5-mg samples from six isothermal experiments ${ }^{10}$ and model predictions. The temperature-dependent thermal conductivity and heat capacity were measured ${ }^{19}$ with a differential scanning calorimeter and a laser flash technique, respectively. The reaction enthalpy was obtained from a differential scanning calorimeter. ${ }^{10}$ Three nonisothermal experiments, not used to estimate kinetic parameters, were simulated to validate the selection of the kinetic parameters for the PUF model.

Various sensitivity and uncertainty analyses were performed using the PolyUrethane Foam (PUF) decomposition model. For high heating rates using fire-like boundary conditions, the most sensitive kinetic parameters were associated with the formation of the secondary polymer. For milder conditions, the reaction zone thickened, and the thermal conductivity, initial density, and reaction enthalpies were shown to be sensitive parameters, as well as the activation energies associated with the destruction of the secondary polymer. In the experiments, a few of the thermocouples used to define the boundary conditions were shown to unexpectedly increase in temperature. Nonuniform burn fronts exposed thermocouple sheaths to the hot boundary. Conduction of heat along the thermocouple sheath to the thermocouple tip may have caused the measured thermocouple to read higher temperatures than the surrounding foam. Heat transfer between the hot tube walls and the thermocouple leads may have also resulted in unaccounted heat transfer to the thermocouple junctions. The uncertainty in the exposed thermocouples is on the order of $100^{\circ} \mathrm{C}$. Future experiments will use more thermocouples to better define the boundary conditions.

A two-dimensional axisymmetric simulation of an unconfined, component-scale experiment was performed using an 11,209 element mesh with the PUF model implemented into COYOTE. ${ }^{1}$ Predictions were made using a grid-independent solution with $0.5-\mathrm{mm}$ by $0.5-\mathrm{mm}$ elements and compared to experimental temperature measurements. Elements were removed from the computational domain whenever the condensed fraction was less than 0.01 , and view factors were recomputed. Good agreement was obtained between temperature measurements and predictions. The shape of the regression front measured with X-ray imaging was also in agreement with the predicted shape of the burning front. Although the agreement between the predicted and measured temperatures was not exact, the temperatures were within experimental and computational uncertainty.

The PUF model describes polyurethane foam decomposition on a more fundamental basis than traditional first order global reaction schemes. In the PUF model, the specific bonds involved in polymer degradation have been 
Submitted to Polymer Degradation and Stability

identified and are used in the reaction scheme. Every broken bond does not guarantee gas evolution from the decomposing polymer. The distribution of gases evolving during decomposition are resolved using percolation theory coupled to a simple vapor-liquid equilibrium model. The agreement between the PUF model predictions of mass loss associated with TGA results over a broad range of heating rates have been acceptable, as well as predictions of foam decomposition in a large-scale experiment utilizing high heating rates. However, neither of these experiments were totally confined, and the decomposition gases were allowed to leave the system. Secondary reactions of the decomposition gases with the reacting solid are expected to be important for confined systems. Furthermore, the PUF model does not consider mass transport limitations or mechanical effects such as thermal expansion which becomes important when large foam samples are exposed to low heat fluxes. Confined decomposition of rigid polyurethane foam will be addressed in future work.

\section{ACKNOWLEDGEMENTS}

The authors gratefully acknowledge the technical assistance provided by J. H. Bentz, W. Gill, L. L. Humphries, and J. G. Pantuso (scale-up experiments), T. A. Ulibarri and T. Neet (provided foam samples and synthesis details), R. S. Saunders (polymer structures and synthesis of model compounds for IR analysis), T. T. Borek (desorption tube analysis), J. N. Castaneda (TGA analysis), and J. D. Kurtz (currently with Proctor and Gamble). Modeling discussions related to percolation theory from D. M. Grant (University of Utah), R. J. Pugmire (University of Utah), and T. H. Fletcher (Brigham Young University) are also appreciated. Discussions regarding uncertainty and sensitivity analysis with M. R. Baer and B. F. Blackwell were helpful. M. S. Eldred helped set up the DAKOTA optimizer used to obtain the activation energies and the object function gradients used in the uncertainty analysis. Comments from internal reviewers at Sandia National Laboratories, A. R. Kerstein, L. M. G. Minier; Jaime Moya, and A. C. Ratzel, are also deeply appreciated. D. K. Gartling performed the three-dimensional calculations shown in Fig. 1, and R. J. Pugmire provided the solid state NMR analysis shown in Fig. 6.C. 


\section{REFERENCES}

1. Gartling, D. K., Hogan, R. E. \& Glass, M. W., "COYOTE - A Finite Element Computer Program for Nonlinear Heat Conduction Problems," SAND94-1173 (theory), SAND94-1179 (user's manual), Sandia National Laboratories, Albuquerque, NM (1998).

2. Solomon, P. R., Hamblen, D. G., Carangelo, R. M., Serio, M. A. \& Deshpande, G. V., Energy \& Fuels, 2 (1988) 405.

3. Grant, D. M., Pugmire, R. J., Fletcher, T. H. \& Kerstein, A. R., Energy \& Fuels, 3 (1989) 175.

4. Fletcher, T. H., Kerstein, A. R., Pugmire, R. J. \& Grant, D. M., Energy \& Fuels, 4 (1990) 54.

5. Fletcher, T. H., Kerstein, A. R., Pugmire, R. J., Solum, M. S. \& Grant, D. M., Energy \& Fuels, 6 (1992) 414.

6. Fisher, M. E. \& Essam, J. W., Journal of Mathematical Physics, 2 (1961) 609.

7. Flory, P. J., Principles of Polymer Chemistry, Cornell University Press., Ithaca, New York (1953) 347.

8. Solum, M. S., Pugmire, R. J. \& Grant, D. M., Energy \& Fuels, 3 (1989)187.

9. Shafizadeh, F., "The Chemistry of Pyrolysis and Combustion," Ch. 13 in The Chemistry of Solid Wood, Advances in Chemistry Series 207, Rowell, R., editor, American Chemical Society, Washington, D. C. (1984).

10. Chu, T. Y., Hobbs, M. L., Erickson, K. L., Ulibarri, T. A., Renlund, A. M., Gill, W., Humphries, L. L. \& Borek, T. T., SAMPE 1999 - 44 $4^{\text {th }}$ International SAMPE Symposium \& Exhibition, Long Beach, CA (1999).

11. Shampine, L. F. \& Watts, H. A., "DEPAC - Design of a User Oriented Package of ODE Solvers," SAND-79-2374, Sandia National Laboratories, Albuquerque, NM (1979).

12. Hobbs, M. L., Erickson, K. E., and Chu, T. Y., Modeling Decomposition of Unconfined Rigid Polyurethane Foam, SAND99-2758, Sandia National Laboratories, Albuquerque, NM (1999).

13. Atkins, P. W., Physical Chemistry, second edition, W. H. Freeman and Company, San Francisco, California (1982).

14. Henley, E. J., \& Seader, J. D., Equilibrium-Stage Separation Operations in Chemical Engineering, John Wiley \& Sons, New York (1981).

15. Shampine, L. F., \& Watts, H. A., "ZEROIN - A Root-Solving Code," SC-TM-70-631, Sandia National Laboratories, Albuquerque, NM (1970).

16. Daubert, T. E., Danner, R. P., Subul, H. M., and Stebbins, C. C., Physical and Thermodynamic Properties of Pure Compounds: Data Compilation, Taylor \& Francis, Bristol, PA (1994). See also Daubert, T. E. and Danner, R. P., Design institute for physical property data, AIChE, Dept. of Chem. Eng., Penn State University (1994).

17. Eldred, M. S., "Optimization Strategies for Complex Engineering Applications," SAND98-0340, UC-705, Sandia National Laboratories, Albuquerque, NM (1998).

18. Wheeler, D. J., Understanding Industrial Experimentation, Second Edition, SPC Press, Inc., Knoxville, TN (1990). See also Notes from "Strategy of Experimentation," copyright held by E. I. duPont de Nemours and Co., Wilmington, Delaware, 19898, in October 1975 (revised edition).

19. Tayler, R. E., Groot, and Ferrier, J., "Thermophysical Properties of a Foam," TPRL 1833, Thermophysical Properties Research Laboratory, Purdue University Research Park, West Lafayette, IN (1997). 


\section{Figure Legends}

Fig. 1 Example calculation of inert components encapsulated in rigid polyurethane foam initially at $100^{\circ} \mathrm{C}$ exposed to a constant flux on the entire exposed surface. Elements were removed when element temperature exceeded $150^{\circ} \mathrm{C}$. Although foam regression is shown as a function of time, a decomposition model was not used for this calculation. Figure used with permission from Gartling. ${ }^{1}$

Fig. 2 Most common chemical structural units and hypothetical chemical structure of rigid polyurethane foam. The graphic symbols are composed of ingredients used to make the specific foam.

Fig. 3 Three most common structural units of a rigid polyurethane foam showing boundaries used to relate chemical structure to a Bethe lattice.

Fig. 4 Kinetic mechanism for decomposition of rigid polyurethane foam with the primary polymer decomposition pathway highlighted.

Fig. 5 Kinetic mechanism for decomposition of rigid polyurethane foam with the secondary polymer decomposition pathway highlighted.

Fig. 6 A) Most probable chemical structure showing two possible weak bonds labeled (1) and (2). B) FTIR spectra showing evidence of urethane bond rupture. C) Solid-state NMR evidence showing change in carbonyl associated with adipic acid and isocyanate structures. Other information available in the NMR data has not been fully analyzed.

Fig. 7 Trimer showing $\sigma, n, s$, and $\tau$.

Fig. 8 5-mer showing sites, bonds, and danglers.

Fig. 9 Comparison of PUF predicted (solid line) and measured (dashed line) solid mass fraction for A) single isothermal TGA, B) dual isothermal TGA, and C) three nonisothermal TGA experiments.

Fig. 10 Comparison of PUF predicted (solid line) and measured (dashed and dotted lines) solid mass fraction for the $20-^{\circ} \mathrm{C} / \mathrm{min}$ TGA experiment. The dashed lines are for a $4.687-\mathrm{mg}$ sample and the dotted line is for a 13.765-mg sample.

Fig. 11 A) Comparison of PUF predicted (solid line) and measured (dashed line) solid mass fraction for a $5-^{\circ} \mathrm{C} / \mathrm{min}$ TGA experiment. B) Predicted population parameters for the $5-^{\circ} \mathrm{C} / \mathrm{min}$ TGA experiment given in A. C) Predicted average volatile molecular weight and various oligomer mass percents for the $5-^{\circ} \mathrm{C} / \mathrm{min}$ TGA experiment given in $\mathrm{A}$.

Fig. 12 Dependence of burn velocity on element size for a one-dimensional model with a radiative boundary condition.

Fig. 13 Grid error calculated from Eq. (50) for a one-dimensional model with flux boundary conditions.

Fig. 14 Schematic of component-scale ambient-pressure vented experiment. ${ }^{9}$

Fig. 15 Foam cylindrical holding cup. The foam (not shown) is $15-\mathrm{cm}$ long and protrudes from the cup.

Fig. $16 \mathrm{X}$-ray image of component-scale experiment after 15-min exposure to an incident flux of $25 \mathrm{~W} / \mathrm{cm}^{2}$.

Fig. 17 Measured cup wall temperatures and bottom cup temperature. ${ }^{10}$ 
Fig. 18 Measured cup wall temperatures and top cup temperature at early times. Note that suspect temperatures Gand 6 should not be hotter than 9 .

Fig. 19 Axisymmetric mesh with 11,209 elements and X-ray of foam in cylindrical holding cup showing 3-mm gap between face of component and foam. The foam is $15-\mathrm{cm}$ long and protrudes from the cup.

Fig. 20 Measured and predicted burn front shape after 10 min exposure to an incident flux $\sim 25 \mathrm{~W} / \mathrm{cm}^{2}, \mathrm{~B}$ ) predicted solid fraction contours, C) measured temperature and density across burn front, and D) predicted temperature and solid fraction across burn front.

Fig. 21 X-ray images (left) and calculated temperature profiles (right) of foam with cup bottom exposed to an incident flux of $\sim 25 \mathrm{~W} / \mathrm{cm}^{2}$.

Fig. 22 Center thermocouple locations and B) midradial thermocouple locations.

Fig. 23 Calculated (solid lines) and measured (dashed lines) temperatures at center thermocouple locations shown in Fig. 25.A.

Fig. 24 Calculated (solid lines) and measured (dashed lines) temperatures at midradial thermocouple locations shown in Fig. 25.B.

Fig. 25 Calculated (lines) and measured (symbols) temperatures of the encapsulated component at the locations indicated in the inset.

Fig. 26 A) Top view of exposed thermocouple and B) side view of exposed thermocouple.

Fig. 27 Predicted transmitted heat flux along the center of the foam.

Fig. 28 Predicted center and midradial burn front velocity. The center and midradial locations are shown in the inset. 
Table1. Mechanism, rate equations, and initial conditions for the PUF model ${ }^{*}$

\begin{tabular}{|c|l|c|l|c|}
\hline Rxn & Mechanism & Species & \multicolumn{1}{|c|}{ Rate Equations } & Initial Conditions \\
\hline & & $L$ & $d \delta / d t=-k_{1} \mathcal{L}-k_{2} \mathcal{L}+k_{3} \delta$ & $\mathcal{L}(0)=\mathcal{L}_{0}$ \\
$(1)$ & $\mathcal{L} \rightarrow \mathrm{c}_{1}+\mathrm{g}_{1}$ & $\mathrm{~L}$ & $d \mathrm{~L} / d t=k_{5} \delta^{2}-k_{6} \mathrm{~L}-k_{7} \mathrm{~L}+k_{8} \mathrm{~L}$ & $\mathrm{~L}(0)=0$ \\
$(2)$ & $\mathcal{L} \rightarrow \delta$ & $\delta$ & $d \delta / d t=k_{2} \mathcal{L}-k_{3} \delta-k_{4} \delta-2 k_{5} \delta^{2}$ & $\delta(0)=1-\mathrm{c}_{0}-\mathcal{L}_{\mathrm{o}}$ \\
$(3)$ & $\delta \rightarrow \mathcal{L}$ & $\mathrm{d}$ & $d \mathrm{~d} / d t=k_{7} \mathrm{~L}-k_{8} \mathrm{~d}-k_{9} \mathrm{~d}$ & $\mathrm{~d}(0)=0$ \\
$(4)$ & $\delta \rightarrow \mathrm{g}_{2}$ & $\mathrm{c}_{1}$ & $d \mathrm{c}_{1} / d t=k_{1} \mathcal{L}$ & $\mathrm{c}_{1}(0)=\mathrm{c}_{\mathrm{o}}$ \\
$(5)$ & $2 \delta \rightarrow \mathrm{L}+\mathrm{g}_{3}$ & $\mathrm{c}_{2}$ & $d \mathrm{c}_{2} / d t=k_{6} \mathrm{~L}$ & $\mathrm{c}_{2}(0)=0$ \\
$(6)$ & $\mathrm{L} \rightarrow \mathrm{c}_{2}+\mathrm{g}_{4}$ & $\mathrm{~g}_{1}$ & $d \mathrm{~g}_{1} / d t=k_{1} \mathcal{L}$ & $\mathrm{g}_{1}(0)=0$ \\
$(7)$ & $\mathrm{L} \rightarrow \mathrm{d}$ & $\mathrm{g}_{2}$ & $d \mathrm{~g}_{2} / d t=k_{4} \delta$ & $\mathrm{g}_{2}(0)=0$ \\
$(8)$ & $\mathrm{d} \rightarrow \mathrm{L}$ & $\mathrm{g}_{3}$ & $d \mathrm{~g}_{3} / d t=k_{5} \delta^{2}$ & $\mathrm{~g}_{3}(0)=0$ \\
$(9)$ & $\mathrm{d} \rightarrow \mathrm{g}_{5}$ & $\mathrm{~g}_{4}$ & $d \mathrm{~g}_{4} / d t=k_{6} \mathrm{~L}$ & $\mathrm{~g}_{4}(0)=0$ \\
& & $\mathrm{~g}_{5}$ & $d \mathrm{~g}_{5} / d t=k_{9} \mathrm{~d}$ & $\mathrm{~g}_{5}(0)=0$ \\
\hline
\end{tabular}

* The concentration $\left(\mu_{\mathrm{ij}}\right)$ and stoichiometric matrices $\left(v_{\mathrm{ij}}\right)$ with the reactions loaded into 9 columns $(j=9)$ and the species loaded into 11 rows $(i=11)$ are written as:

$$
\begin{aligned}
& \begin{array}{lllllllll}
r_{1} & r_{2} & r_{3} & r_{4} & r_{5} & r_{6} & r_{7} & r_{8} & r_{9}
\end{array} \\
& \mathcal{L}\left[\begin{array}{lllllllll}
1 & 1 & 0 & 0 & 0 & 0 & 0 & 0 & 0
\end{array}\right] \\
& \text { L } \quad \begin{array}{lllllllll}
0 & 0 & 0 & 0 & 0 & 1 & 1 & 0 & 0
\end{array}
\end{aligned}
$$

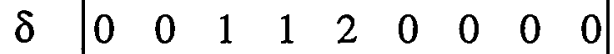

$$
\begin{aligned}
& \text { d } \quad 0 \begin{array}{lllllllll}
0 & 0 & 0 & 0 & 0 & 0 & 0 & 1 & 1
\end{array}
\end{aligned}
$$

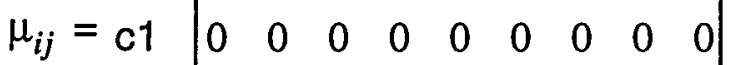

$$
\begin{aligned}
& \text { c2 } \quad 0 \begin{array}{lllllllll}
0 & 0 & 0 & 0 & 0 & 0 & 0 & 0 & 0
\end{array}
\end{aligned}
$$

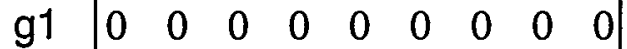

$$
\begin{aligned}
& \text { g2 } \quad 0 \begin{array}{lllllllll}
0 & 0 & 0 & 0 & 0 & 0 & 0 & 0
\end{array} \\
& \text { g3 } \quad 0 \begin{array}{lllllllll}
0 & 0 & 0 & 0 & 0 & 0 & 0 & 0
\end{array} \\
& \text { g4 } 5 \begin{array}{lllllllll}
0 & 0 & 0 & 0 & 0 & 0 & 0 & 0 & 0 \\
0 & 0 & 0 & 0 & 0 & 0 & 0 & 0 & 0
\end{array}
\end{aligned}
$$

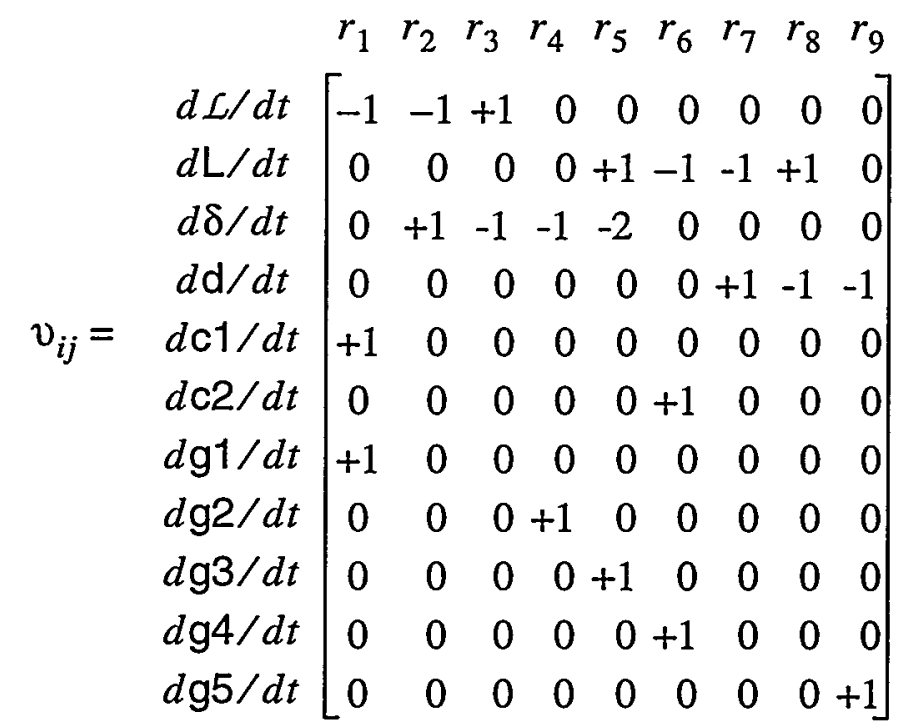


Table 2. PUF model parameters with estimated values for sensitivity analysis."

\begin{tabular}{|c|c|c|c|c|}
\hline $\begin{array}{l}\text { Variable } \\
\text { Symbol }\end{array}$ & Description & $\begin{array}{c}\text { Nominal } \\
\text { Value }\end{array}$ & Low Value (t) & High Value (-) \\
\hline$P_{o}$ & Initial bond population ${ }^{\dagger}$ & 0.85 & 0.8 & 0.9 \\
\hline$c_{o}$ & Initial strong bond population $^{\dagger}$ & 0.10 & 0.05 & 0.15 \\
\hline$\sigma+1$ & Coordination number & 2.8 & 2.1 & 3.0 \\
\hline$M_{c}$ & Site molecular weight ${ }^{\ddagger}$ & $125 \mathrm{~g} / \mathrm{mol}$ & $104 \mathrm{~g} / \mathrm{mol}$ & $131 \mathrm{~g} / \mathrm{mol}$ \\
\hline$M_{b}$ & Bridge molecular weight ${ }^{\ddagger}$ & $161 \mathrm{~g} / \mathrm{mol}$ & $155 \mathrm{~g} / \mathrm{mol}$ & $163 \mathrm{~g} / \mathrm{mol}$ \\
\hline$M_{g 3}$ & Molecular weight of $g_{3}$ & $44 \mathrm{~g} / \mathrm{mol}$ & $44 \mathrm{~g} / \mathrm{mol}$ & $44 \mathrm{~g} / \mathrm{mol}$ \\
\hline$P$ & Pressure $^{t}$ & $83 \mathrm{KPa}$ & $82 \mathrm{KPa}$ & $84 \mathrm{KPa}$ \\
\hline$\overline{E_{1}}$ & Activation Energy for reaction $1^{\dagger}$ & $53400 . \mathrm{cal} / \mathrm{mol}$ & $0.9 \times E_{1}$ & $1.1 \times E_{l}$ \\
\hline$E_{2}$ & Activation Energy for reaction $2^{\dagger}$ & $44580 . \mathrm{cal} / \mathrm{mol}$ & $0.9 \times E_{2}$ & $1.1 \times E_{2}$ \\
\hline$E_{3}$ & Activation Energy for reaction $3^{\dagger}$ & 42520. $\mathrm{cal} / \mathrm{mol}$ & $0.9 \times E_{3}$ & $1.1 \times E_{3}$ \\
\hline$E_{4}$ & Activation Energy for reaction $4^{\dagger}$ & 45900. cal $/ \mathrm{mol}$ & $0.9 \times E_{4}$ & $1.1 \times E_{4}$ \\
\hline$E_{5}$ & Activation Energy for reaction $5^{\dagger}$ & 44600. $\mathrm{cal} / \mathrm{mol}$ & $0.9 \times E_{5}$ & $1.1 \times E_{5}$ \\
\hline$E_{6}$ & Activation Energy for reaction $6^{\dagger}$ & $58060 . \mathrm{cal} / \mathrm{mol}$ & $0.9 \times E_{6}$ & $1.1 \times E_{6}$ \\
\hline$E_{7}$ & Activation Energy for reaction $7^{\dagger}$ & $51850 . \mathrm{cal} / \mathrm{mol}$ & $0.9 \times E_{7}$ & $1.1 \times E_{7}$ \\
\hline$E_{8}$ & Activation Energy for reaction $8^{\dagger}$ & $52830 . \mathrm{cal} / \mathrm{mol}$ & $0.9 \times E_{8}$ & $1.1 \times E_{8}$ \\
\hline$E_{9}$ & Activation Energy for reaction $9^{\dagger}$ & 56660. $\mathrm{cal} / \mathrm{mol}$ & $0.9 \times E_{9}$ & $1.1 \times E_{9}$ \\
\hline
\end{tabular}

* All frequency factors, $A$, were assumed to be equal to $3.0 \times 10^{15} \mathrm{~s}^{-1}$.

$\dagger$ High and low values guessed.

$\ddagger$ High and low values from the $10 \%$ and $60 \%$ most probable chemical structure. 
Table 3. Molecular weight and coordination number of common foam structural units

\begin{tabular}{|c|r|c|r|}
\hline Structure $^{*}$ & \multicolumn{1}{|c|}{$\boldsymbol{M}_{\boldsymbol{a}}, \mathrm{g} / \mathrm{moll}$} & $\boldsymbol{M}_{\boldsymbol{b}}, \mathrm{g} / \mathbf{m o l}$ & $\sigma+\mathbf{1}$ \\
\hline $60 \%$ most probable & 131 & {$[(4 \times 176)+112] / 5=163$} & 3 \\
$20 \%$ most probable & $(104+131) / 2=118$ & {$[(3 \times 176)+112] / 4=160$} & $(2+3) / 2=2.5$ \\
$10 \%$ most probable & 104 & {$[(2 \times 176)+112] / 3=155$} & 2 \\
\hline
\end{tabular}

* See Fig. 3 for the boundaries used to decompose the most probable structural units into bridges used to determine $M_{B}$ and sites used to determine $M_{A}$ as well as $\sigma+1$. 
Table 4. Calculated factor effects from Plackett Burman Analysis*

\begin{tabular}{|c|c|c|c|c|c|c|c|c|c|c|c|c|c|c|c|}
\hline Time, s & $P_{0}$ & $c_{0}$ & $\sigma+1$ & $M_{c}$ & $M_{b}$ & $P$ & $E_{l}$ & $E_{2}$ & $E_{3}$ & $E_{4}$ & $E_{5}$ & $E_{6}$ & $E_{7}$ & $E_{8}$ & $E_{g}$ \\
\hline 480 & 1.25 & 0.57 & $-3.18^{\dagger}$ & 0.37 & 0.75 & -0.75 & -0.12 & -0.82 & -0.80 & 0.62 & $3.18^{\dagger}$ & -0.80 & 0.37 & -0.82 & -1.25 \\
\hline 540 & $2.29^{\dagger}$ & 0.69 & $-3.34^{\dagger}$ & -0.20 & 1.06 & -1.06 & 0.47 & -0.96 & -1.13 & 0.76 & $3.34^{\dagger}$ & -1.13 & -0.20 & -0.96 & $-2.29^{\dagger}$ \\
\hline 600 & $4.41^{\dagger}$ & 0.83 & $-2.30^{\dagger}$ & -0.58 & $2.52^{\dagger}$ & 0.32 & 0.59 & 0.78 & -0.37 & 1.40 & $7.59^{\dagger}$ & -1.55 & -0.59 & -0.64 & -1.70 \\
\hline 660 & $2.59^{\dagger}$ & 1.23 & $-2.69^{\dagger}$ & -1.02 & 0.51 & 0.39 & -0.16 & 1.23 & -1.32 & $2.42^{\dagger}$ & $5.48^{\dagger}$ & -1.19 & -0.73 & 0.61 & -1.92 \\
\hline 720 & 0.29 & 0.82 & $-2.35^{\dagger}$ & -0.55 & -0.31 & -0.29 & -1.71 & $3.16^{\dagger}$ & $-3.31^{\dagger}$ & $2.58^{\dagger}$ & $4.65^{\dagger}$ & -1.97 & 0.42 & $2.97^{\dagger}$ & -1.16 \\
\hline 780 & -0.92 & 1.00 & $-2.77^{\dagger}$ & 0.45 & -0.95 & -0.48 & -1.94 & $4.43^{\dagger}$ & $-3.25^{\dagger}$ & $3.56^{\dagger}$ & $4.06^{\dagger}$ & $-2.80^{\dagger}$ & 0.42 & $3.44^{\dagger}$ & 0.06 \\
\hline 840 & -0.64 & 1.08 & -0.10 & 0.66 & 0.34 & -1.40 & -0.40 & $3.94^{\dagger}$ & -0.19 & $4.19^{\dagger}$ & 1.20 & -0.68 & 0.84 & 1.16 & 0.96 \\
\hline 900 & -0.80 & 0.60 & 0.26 & 0.48 & 0.38 & -1.12 & 0.17 & $2.62^{\dagger}$ & 1.02 & $2.75^{\dagger}$ & -0.04 & -0.10 & 0.53 & -0.15 & 0.71 \\
\hline 960 & -0.75 & 0.33 & 0.34 & 0.47 & 0.48 & -1.04 & 0.42 & $2.41^{\dagger}$ & 1.09 & $3.02^{\dagger}$ & 0.03 & -0.11 & 0.58 & -0.15 & 0.72 \\
\hline 1020 & -0.93 & 0.03 & 0.80 & 0.73 & 0.82 & -0.91 & 1.68 & 2.10 & 0.93 & $4.13^{\dagger}$ & -0.10 & -0.51 & 0.28 & -0.47 & 0.56 \\
\hline 1080 & -0.89 & -0.69 & 1.59 & 0.40 & -0.19 & -0.89 & $2.25^{\dagger}$ & 0.89 & 0.19 & $5.96^{\dagger}$ & -0.02 & -0.26 & 0.27 & -1.23 & 0.56 \\
\hline 1140 & -0.46 & -1.19 & 1.14 & -0.04 & -1.38 & -0.27 & 1.73 & 1.02 & -0.98 & $5.06^{\dagger}$ & -0.49 & 0.55 & 0.68 & -1.50 & 0.99 \\
\hline 1200 & -0.39 & -1.25 & 0.18 & 0.00 & -1.44 & -0.01 & 0.90 & 1.20 & -1.10 & $3.53^{\dagger}$ & -0.90 & 1.02 & 0.31 & -0.64 & 1.00 \\
\hline 1260 & -0.42 & -0.87 & -0.22 & -0.11 & -1.07 & 0.08 & 0.29 & 1.00 & -1.01 & $2.15^{\dagger}$ & -1.10 & 1.27 & -0.15 & -0.05 & 0.84 \\
\hline 1320 & -0.51 & -0.81 & -0.05 & -0.17 & -1.03 & 0.20 & 0.20 & 1.24 & -1.09 & 1.91 & -1.13 & 1.37 & -0.22 & -0.12 & 0.92 \\
\hline 1380 & -0.45 & -0.90 & 0.45 & -0.15 & -0.78 & 0.68 & -0.25 & 1.81 & -0.89 & 1.49 & -1.14 & 1.29 & -0.44 & -0.63 & 1.38 \\
\hline 1440 & -0.51 & -0.70 & 0.94 & 0.31 & -0.75 & 1.18 & -1.02 & $2.26^{\dagger}$ & -0.65 & 1.06 & -1.46 & 1.51 & -0.28 & -1.15 & 1.94 \\
\hline 1500 & -0.59 & -0.39 & 1.23 & 0.85 & -0.71 & 1.45 & -1.43 & $2.64^{\dagger}$ & -0.82 & 0.79 & -1.85 & 1.65 & -0.12 & -1.48 & 2.09 \\
\hline
\end{tabular}

* Confidence interval (double-sided probability point of t-distribution): $80 \%(1.33) \quad 90 \%(2.13) \quad 95 \%(2.78) \quad 98 \%(3.75) \quad 99 \%(4.60)$

†Significant factor effects based on $90 \%$ confidence interval using four degrees of freedom. 
Table 5. Thermophysical properties of foam

\begin{tabular}{|c|c|c|}
\hline $\begin{array}{l}\text { Variable } \\
\text { Symbol }\end{array}$ & Description & $\begin{array}{l}\text { Nominal } \\
\text { Value }\end{array}$ \\
\hline$\rho_{o}$ & Initial foam density, $\mathrm{g} / \mathrm{cm}^{3}$ & 0.353 \\
\hline$k_{f}$ & $\begin{array}{l}\text { Thermal conductivity, }{ }^{5} \mathrm{cal} / \mathrm{s}-\mathrm{cm}-\mathrm{K} \\
23^{\circ} \mathrm{C} \\
50^{\circ} \mathrm{C} \\
100^{\circ} \mathrm{C} \\
150^{\circ} \mathrm{C} \\
200^{\circ} \mathrm{C} \\
250^{\circ} \mathrm{C}\end{array}$ & $\begin{array}{l}1.4 \times 10^{-4} \\
1.5 \times 10^{-4} \\
1.6 \times 10^{-4} \\
1.8 \times 10^{-4} \\
2.0 \times 10^{-4} \\
2.2 \times 10^{-4}\end{array}$ \\
\hline$c_{p f}$ & $\begin{array}{l}\text { Heat capacity, }{ }^{5} \mathrm{cal} / \mathrm{g}-\mathrm{K} \\
23^{\circ} \mathrm{C} \\
50^{\circ} \mathrm{C} \\
100^{\circ} \mathrm{C} \\
150^{\circ} \mathrm{C} \\
200^{\circ} \mathrm{C} \\
250^{\circ} \mathrm{C}\end{array}$ & $\begin{array}{l}0.303 \\
0.324 \\
0.358 \\
0.440 \\
0.475 \\
0.526\end{array}$ \\
\hline
\end{tabular}


Table 6. Uncertainty Analysis for Radiation Boundary Condition ${ }^{*}$

\begin{tabular}{|c|c|c|c|c|}
\hline$i$ & $\zeta_{i}$ & $d V / d \zeta_{i}$ & $\Delta \zeta_{i}$ & $\left(\Delta \zeta_{i} \frac{d V}{d \zeta_{i}}\right)^{2}, \mathrm{~cm}^{2} / \mathrm{min}^{2}$ \\
\hline$\rho_{0}$ & $0.353 \mathrm{~g} / \mathrm{cm}^{3}$ & $9.93 \times 10^{-1}$ & $0.01 \times \zeta_{i}$ & $0.0000123(0.1 \%)$ \\
\hline$k_{f}$ & 1.0 & $2.93 \times 10^{0}$ & $0.01 \times \zeta_{i}$ & $0.0008601(4.4 \%)$ \\
\hline$c_{p f}$ & 1.0 & $1.90 \times 10^{0}$ & $0.01 \times \zeta_{i}$ & $0.0003626(1.9 \%)$ \\
\hline$T_{o}$ & $300 \mathrm{~K}$ & $-8.59 \times 10^{-3}$ & $0.01 \times \zeta_{i}$ & $0.0006648(3.4 \%)$ \\
\hline$E_{l}$ & $53400 \mathrm{cal} / \mathrm{mol}$ & $-1.94 \times 10^{-5}$ & $0.01 \times \zeta_{i}$ & $0.0001071(0.6 \%)$ \\
\hline$\overline{E_{2}}$ & $44580 \mathrm{cal} / \mathrm{mol}$ & $-9.90 \times 10^{-5}$ & $0.01 \times \zeta_{i}$ & $0.0019478(10.0 \%)$ \\
\hline$E_{3}$ & $42520 \mathrm{cal} / \mathrm{mol}$ & $7.33 \times 10^{-6}$ & $0.01 \times \zeta_{i}$ & $0.0000097(0.0 \%)$ \\
\hline$\overline{E_{4}}$ & $45900 \mathrm{cal} / \mathrm{mol}$ & $-2.30 \times 10^{-4}$ & $0.01 \times \zeta_{i}$ & $0.0111401(57.3 \%)$ \\
\hline$E_{5}$ & $44600 \mathrm{cal} / \mathrm{mol}$ & $1.28 \times 10^{-4}$ & $0.01 \times \zeta_{i}$ & $0.0032844(16.9 \%)$ \\
\hline$E_{6}$ & $58060 \mathrm{cal} / \mathrm{mol}$ & $2.79 \times 10^{-5}$ & $0.01 \times \zeta_{i}$ & $0.0002632(1.4 \%)$ \\
\hline$E_{7}$ & $51850 \mathrm{cal} / \mathrm{mol}$ & $2.32 \times 10^{-5}$ & $0.01 \times \zeta_{i}$ & $0.0001449(0.7 \%)$ \\
\hline$E_{8}$ & $52830 \mathrm{cal} / \mathrm{mol}$ & $-2.31 \times 10^{-5}$ & $0.01 \times \zeta_{i}$ & $0.0001492(0.8 \%)$ \\
\hline$E_{9}$ & $56660 \mathrm{cal} / \mathrm{mol}$ & $-3.10 \times 10^{-5}$ & $0.01 \times \zeta_{i}$ & $0.0003085(1.6 \%)$ \\
\hline$h_{r f}$ & $-35 \mathrm{cal} / \mathrm{cm}^{3}$ & $3.49 \times 10^{-2}$ & $0.01 \times \zeta_{i}$ & $0.0001492(0.8 \%)$ \\
\hline \multirow[t]{2}{*}{$\varepsilon$} & \multirow[t]{2}{*}{0.8} & \multirow[t]{2}{*}{$7.56 \times 10^{-1}$} & \multirow[t]{2}{*}{$0.01 \times \zeta_{i}$} & $0.0000366(0.2 \%)$ \\
\hline & & & & $\Sigma=0.01944(100 \%)$ \\
\hline
\end{tabular}

*The burn velocity for a radiation boundary, with source temperature of $1,000^{\circ} \mathrm{C}$, is $0.97 \pm 0.14 \mathrm{~cm} / \mathrm{min}$. 
Table 7. Uncertainty Analysis for Constant Flux Boundary Condition ${ }^{*}$

\begin{tabular}{|c|c|c|c|c|}
\hline$i$ & $\zeta_{i}$ & $d V / d \zeta_{i}$ & $\Delta \zeta_{i}$ & $\left(\Delta \zeta_{i} \frac{d V}{d \zeta_{i}}\right)^{2}, \mathrm{~cm}^{2} / \mathrm{min}^{2}$ \\
\hline$\rho_{o}$ & $0.353 \mathrm{~g} / \mathrm{cm}^{3}$ & $-4.51 \times 10^{0}$ & $0.01 \times \zeta_{i}$ & $0.0002540(11.1 \%)$ \\
\hline$k_{f}$ & 1.0 & $1.09 \times 10^{0}$ & $0.01 \times \zeta_{i}$ & $0.0001178(5.1 \%)$ \\
\hline$c_{p f}$ & 1.0 & $1.48 \times 10^{-1}$ & $0.01 \times \zeta_{i}$ & $0.0000022(0.1 \%)$ \\
\hline$T_{o}$ & $300 \mathrm{~K}$ & $-3.09 \times 10^{-3}$ & $0.01 \times \zeta_{i}$ & $0.0000862(3.8 \%)$ \\
\hline$E_{l}$ & $53400 \mathrm{cal} / \mathrm{mol}$ & $2.53 \times 10^{-6}$ & $0.01 \times \zeta_{i}$ & $0.0000018(0.1 \%)$ \\
\hline$E_{2}$ & $44580 \mathrm{cal} / \mathrm{mol}$ & $-1.68 \times 10^{-5}$ & $0.01 \times \zeta_{i}$ & $0.0000561(2.5 \%)$ \\
\hline$E_{3}$ & $42520 \mathrm{cal} / \mathrm{mol}$ & $-6.30 \times 10^{-6}$ & $0.01 \times \zeta_{i}$ & $0.0000072(0.3 \%)$ \\
\hline$E_{4}$ & $45900 \mathrm{cal} / \mathrm{mol}$ & $-5.20 \times 10^{-6}$ & $0.01 \times \zeta_{i}$ & $0.0000057(0.2 \%)$ \\
\hline$E_{5}$ & $44600 \mathrm{cal} / \mathrm{mol}$ & $-4.16 \times 10^{-5}$ & $0.01 \times \zeta_{i}$ & $0.0003446(15.1 \%)$ \\
\hline$E_{6}$ & $58060 \mathrm{cal} / \mathrm{mol}$ & $-2.88 \times 10^{-5}$ & $0.01 \times \zeta_{i}$ & $0.0002800(12.2 \%)$ \\
\hline$E_{7}$ & $51850 \mathrm{cal} / \mathrm{mol}$ & $7.95 \times 10^{-6}$ & $0.01 \times \zeta_{i}$ & $0.0000170(0.7 \%)$ \\
\hline$E_{8}$ & $52830 \mathrm{cal} / \mathrm{mol}$ & $-3.61 \times 10^{-5}$ & $0.01 \times \zeta_{i}$ & $0.0003627(15.9 \%)$ \\
\hline$E_{9}$ & $56660 \mathrm{cal} / \mathrm{mol}$ & $3.06 \times 10^{-5}$ & $0.01 \times \zeta_{i}$ & $0.0003012(13.2 \%)$ \\
\hline$h_{r f}$ & $-35 \mathrm{cal} / \mathrm{cm}^{3}$ & $6.07 \times 10^{-2}$ & $0.01 \times \zeta_{i}$ & $0.0004516(19.7 \%)$ \\
\hline & & & & $\Sigma=0.002288(100 \%)$ \\
\hline
\end{tabular}

*The burn velocity for a constant flux of $0.5 \mathrm{cal} / \mathrm{s}-\mathrm{cm}^{2}$ is $0.26 \pm 0.05 \mathrm{~cm} / \mathrm{min}$. 

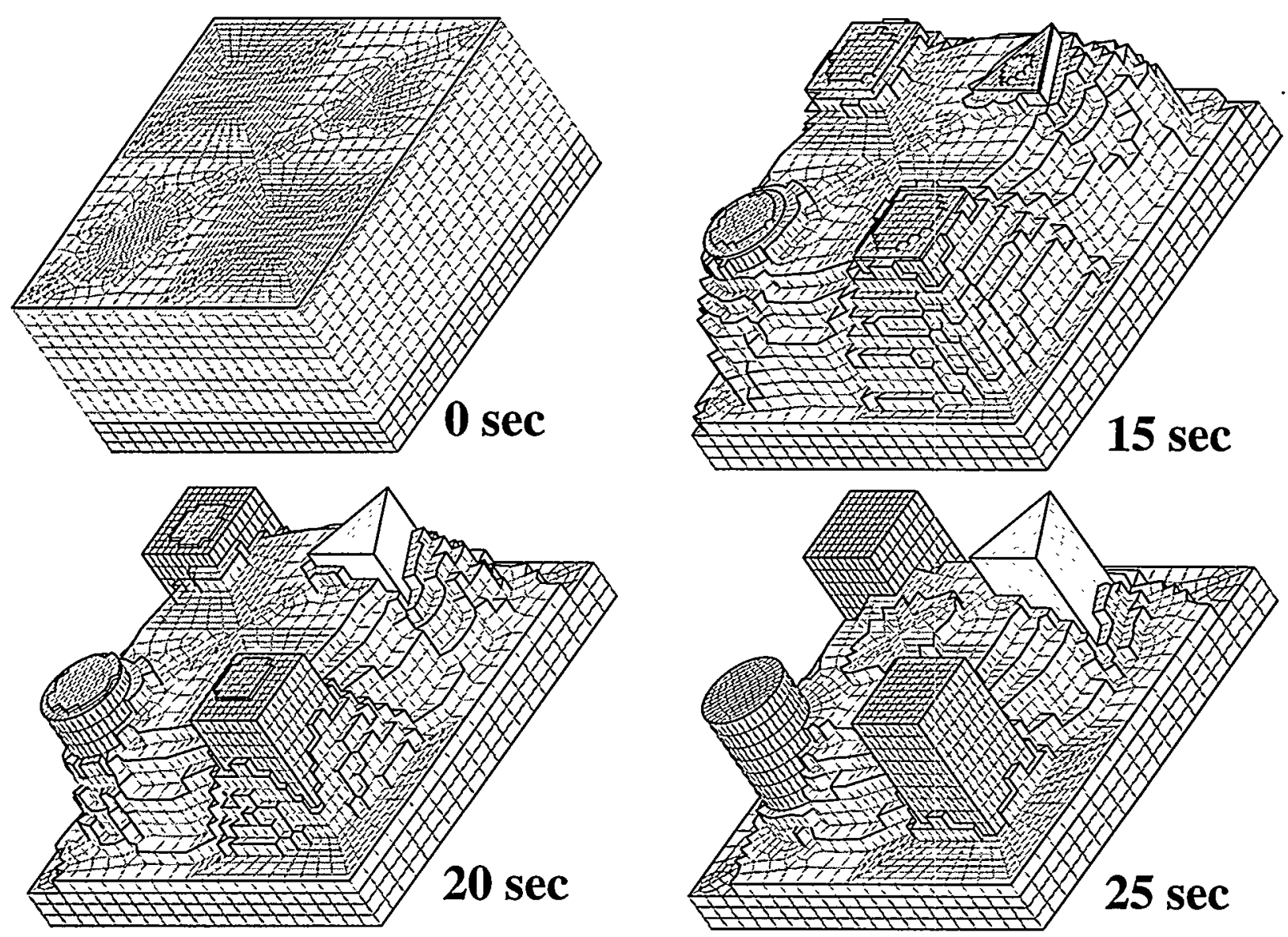

Fig. 1 


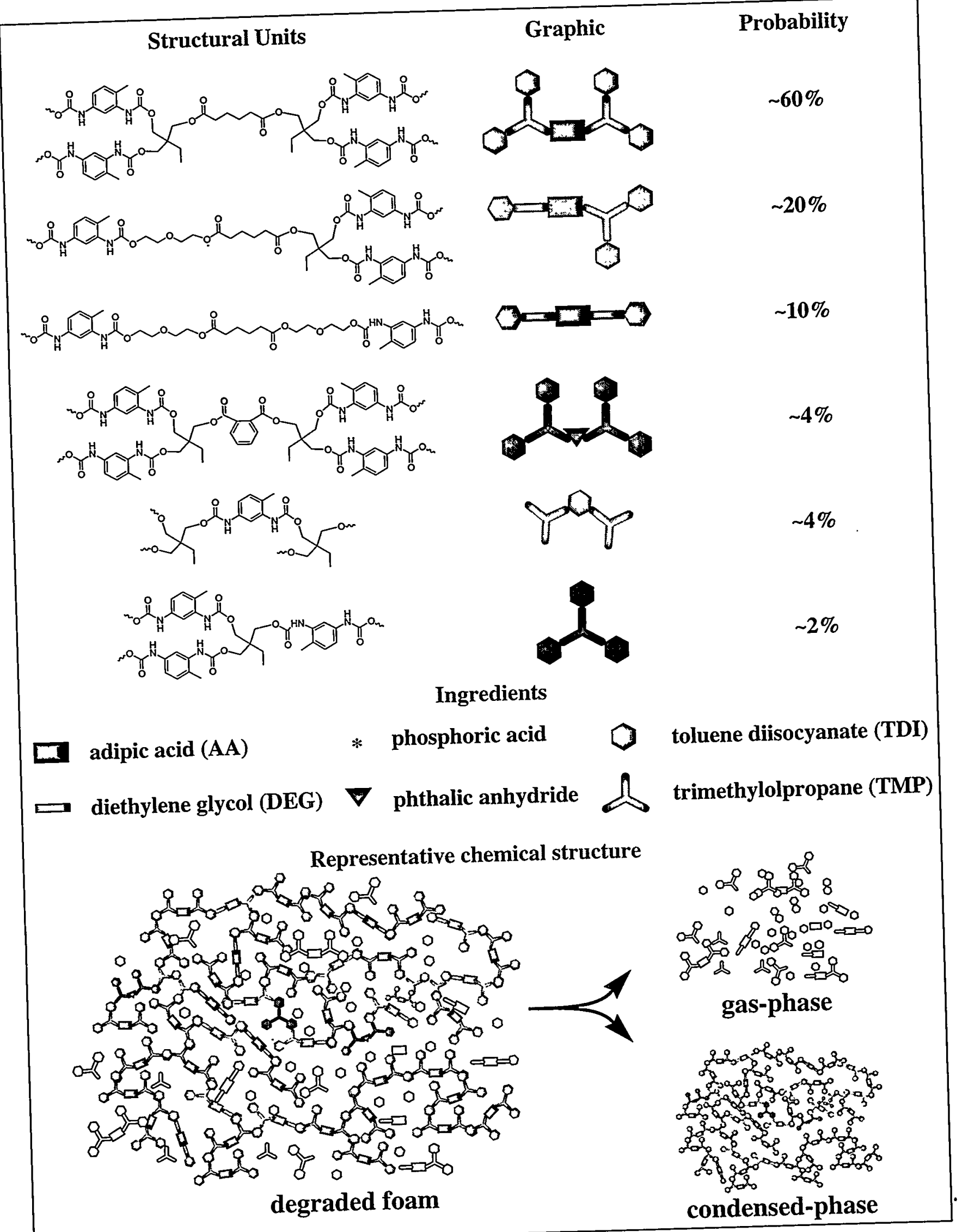

Fig. 2 
A) $60 \%$ most probable structural unit of a rigid polyurethane foam

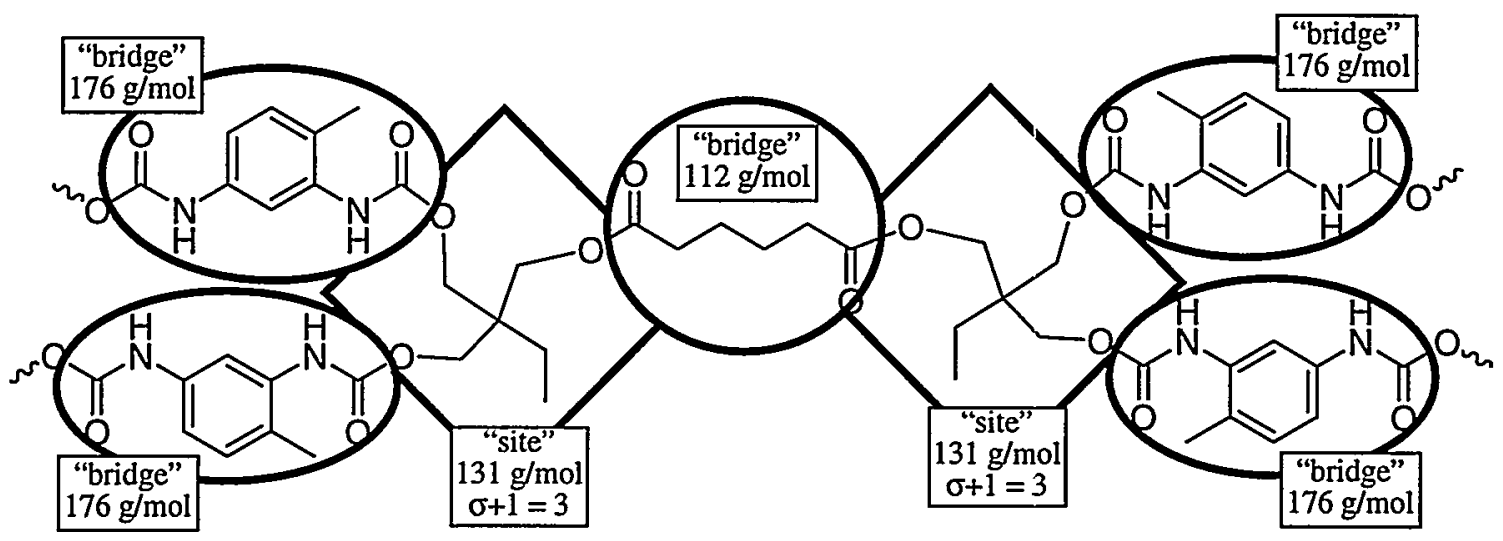

B) $20 \%$ most probable structural unit of a rigid polyurethane foam

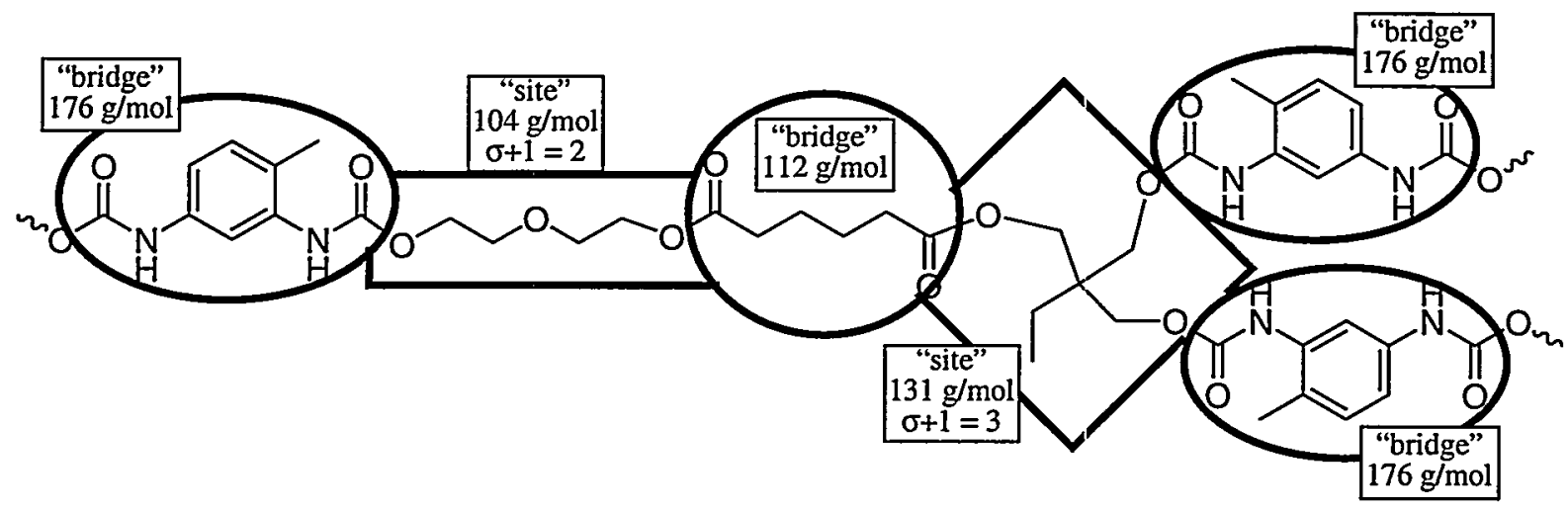

C) $10 \%$ most probable structural unit of a rigid polyurethane foam

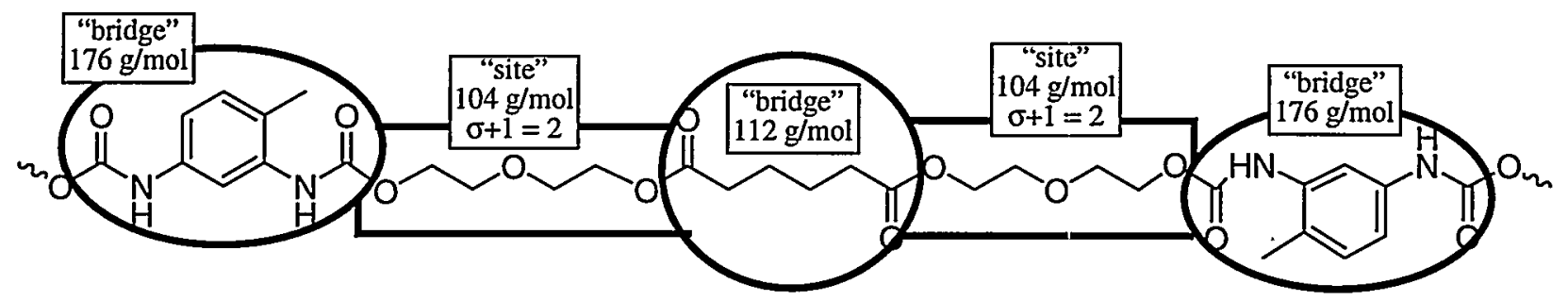

Fig. 3 


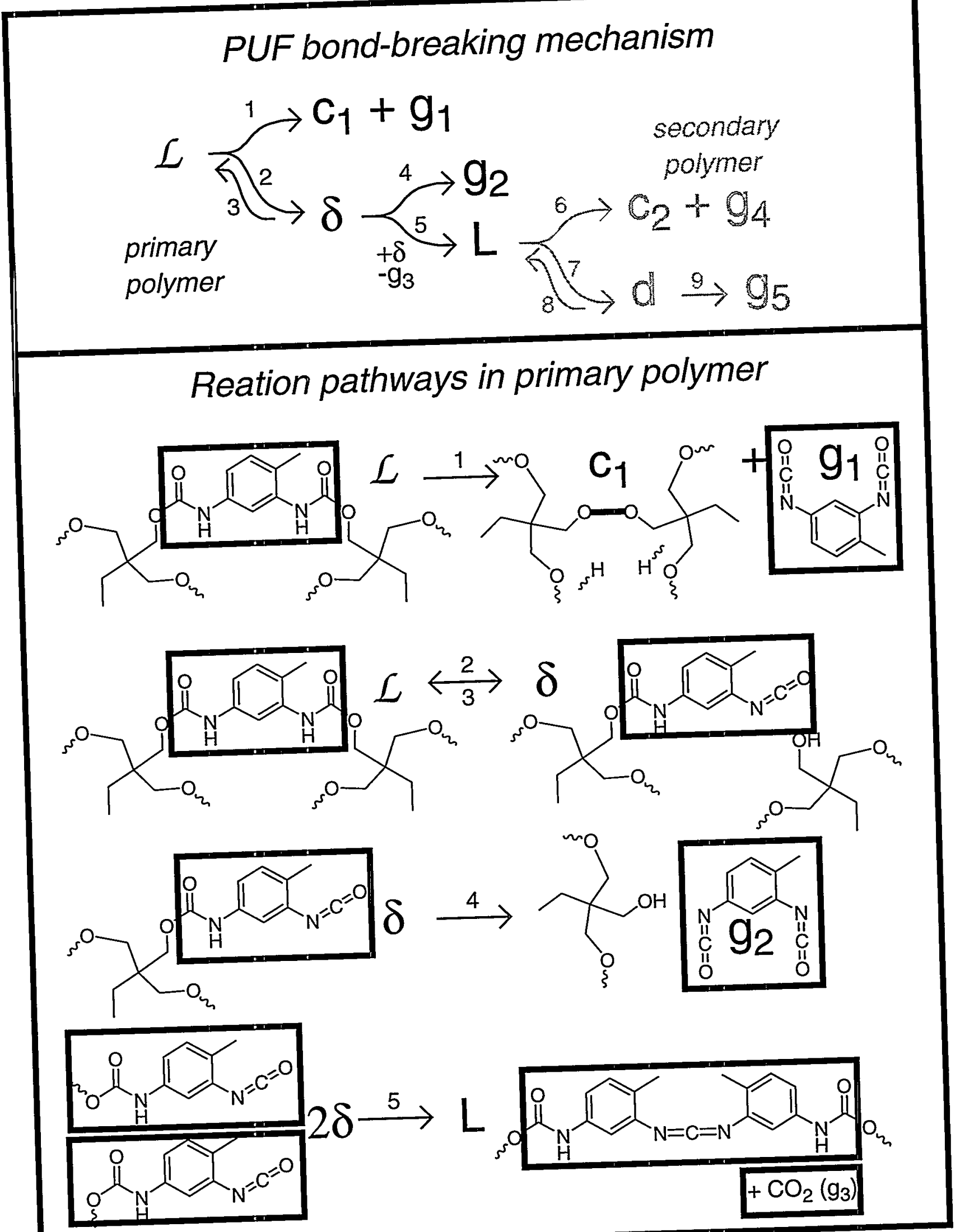

Fig. 4 
PUF bond-breaking mechanism

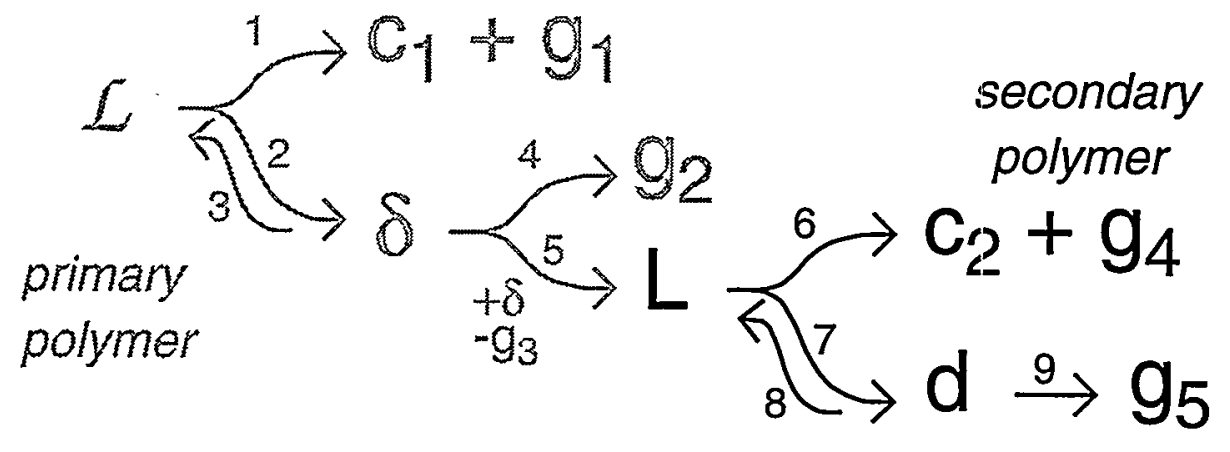

Reation pathways in secondary polymer

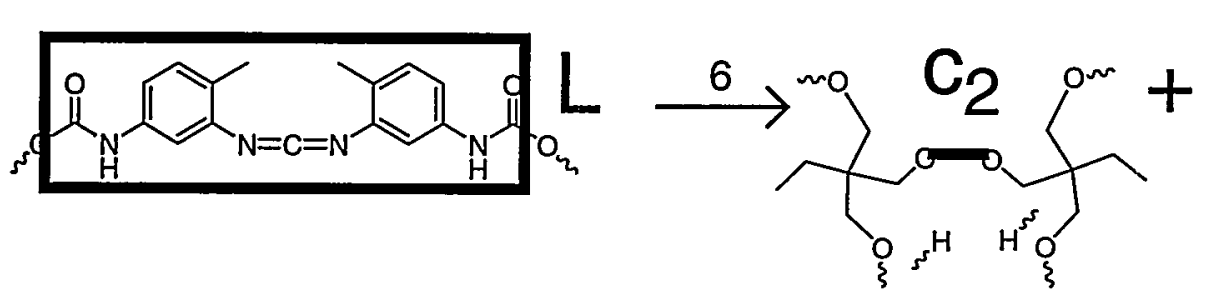

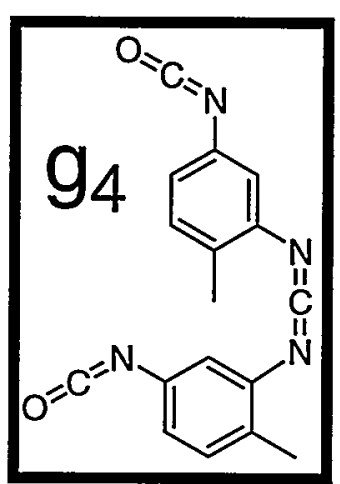

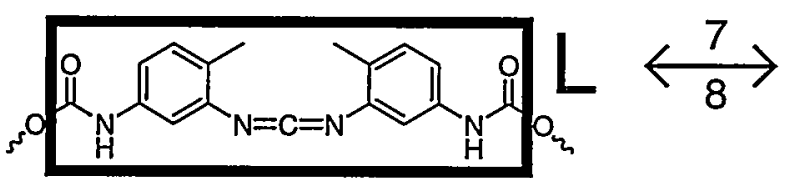

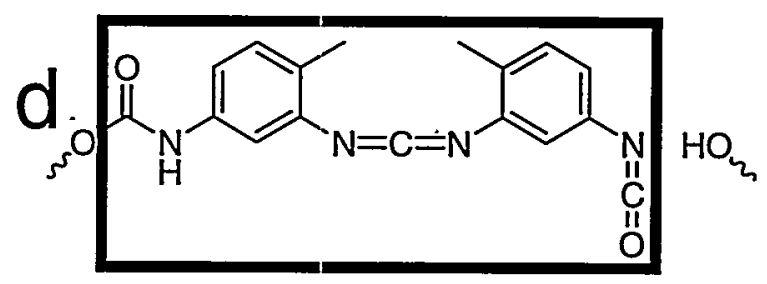

so

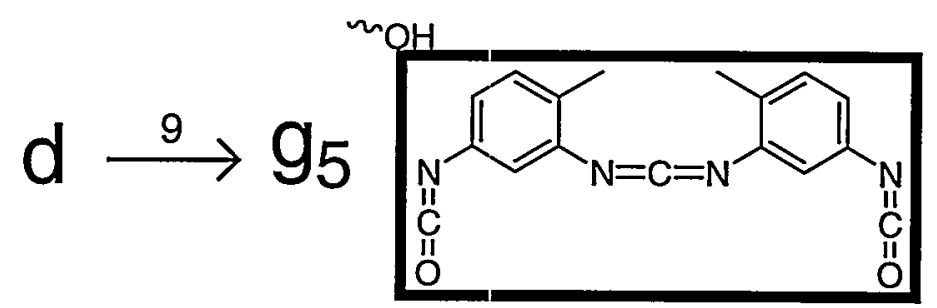

Fig. 5 

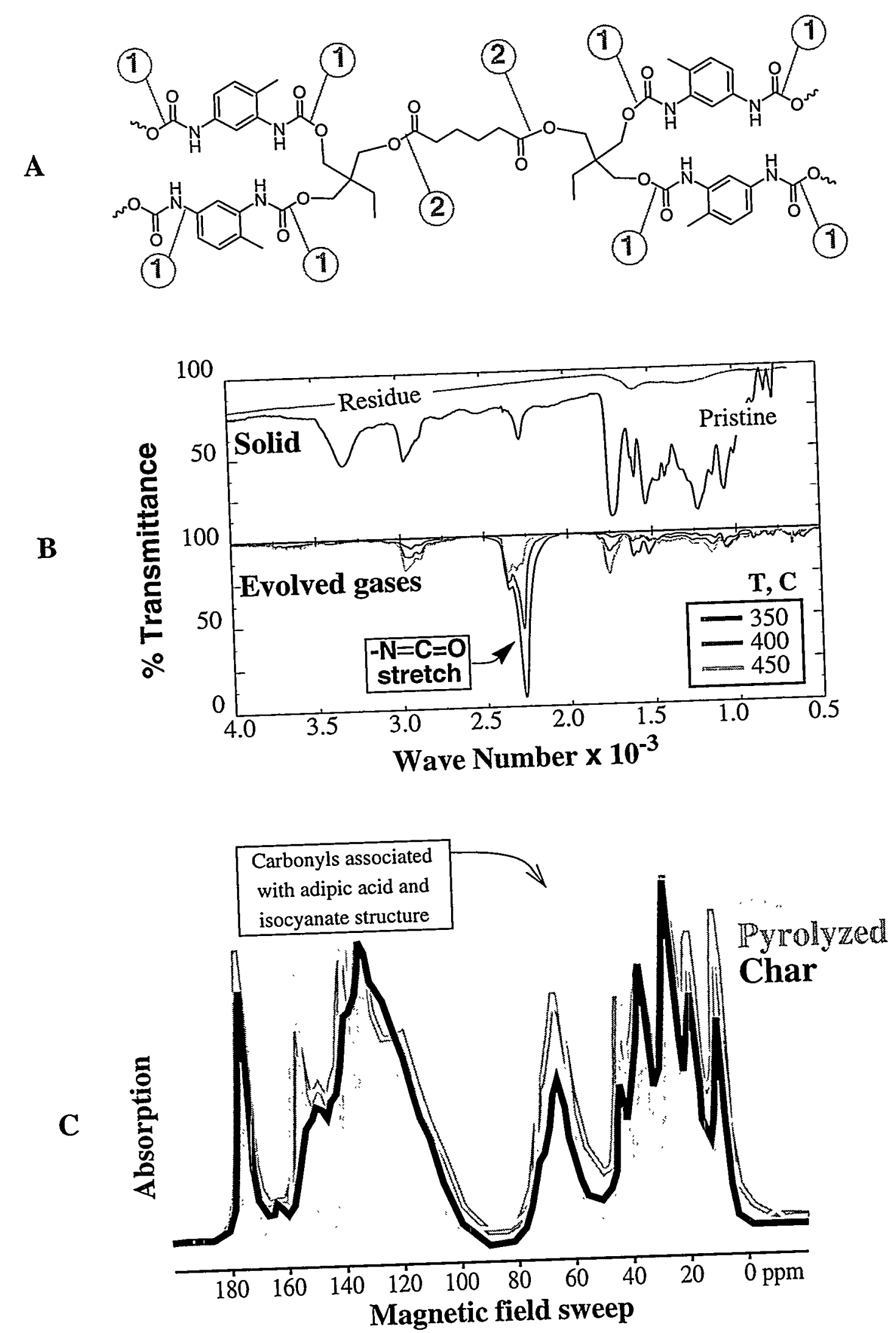

Fig. 6 


\section{Trimer (oligomer with 3 sites)}

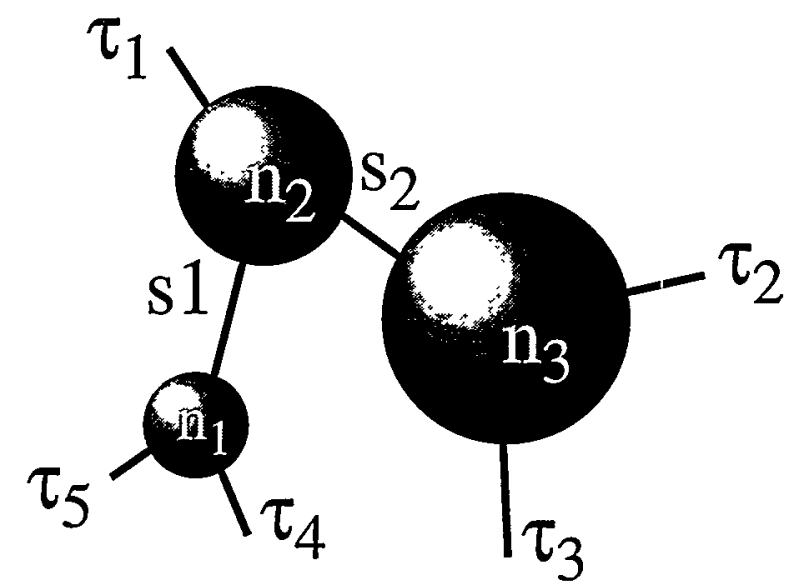

$\sigma=2$ (coordination number -1 )

$n=3$ sites (spheres)

$s=2$ bonds (connecting spheres)

$\tau=5$ broken bonds on perimeter

Fig. 7 


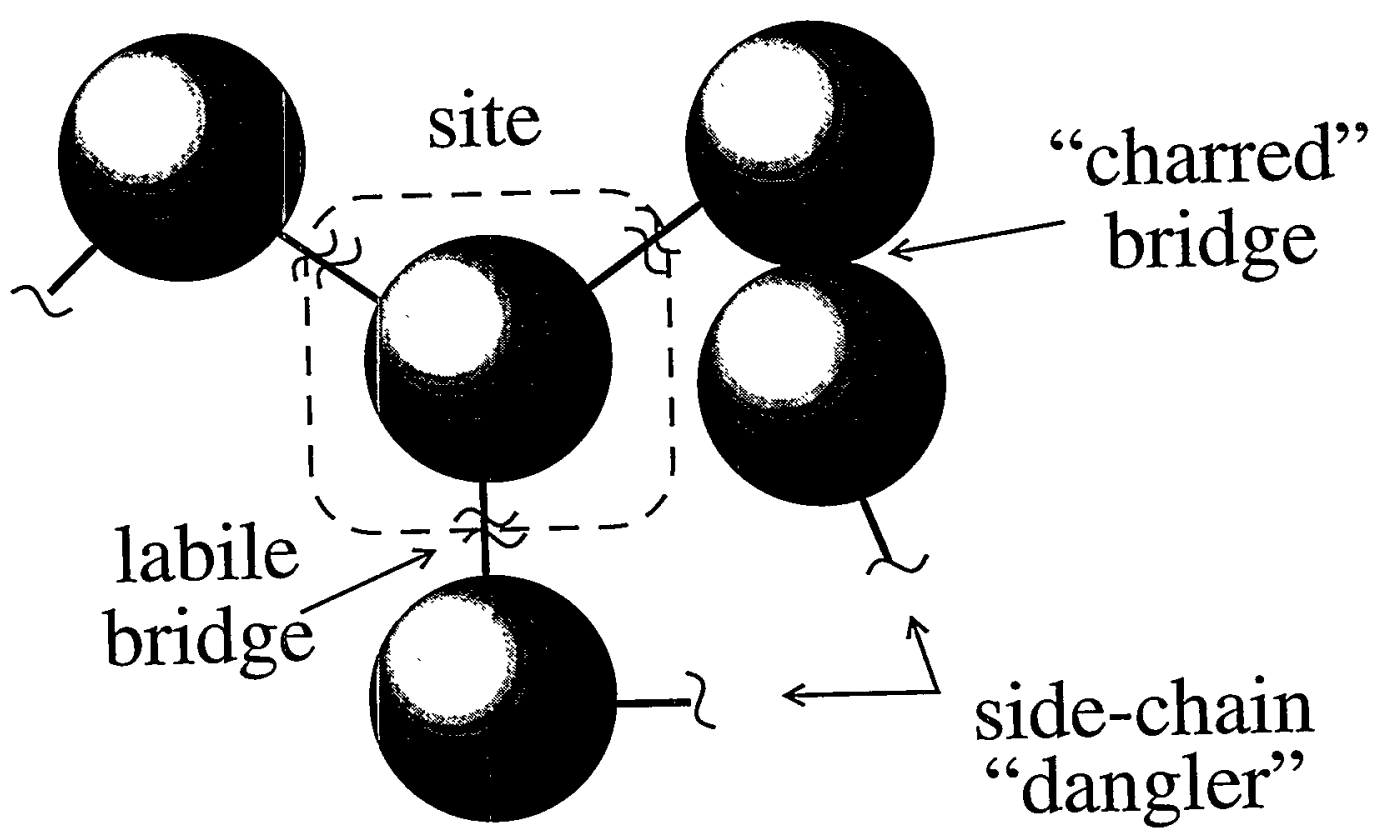

Fig. 8 

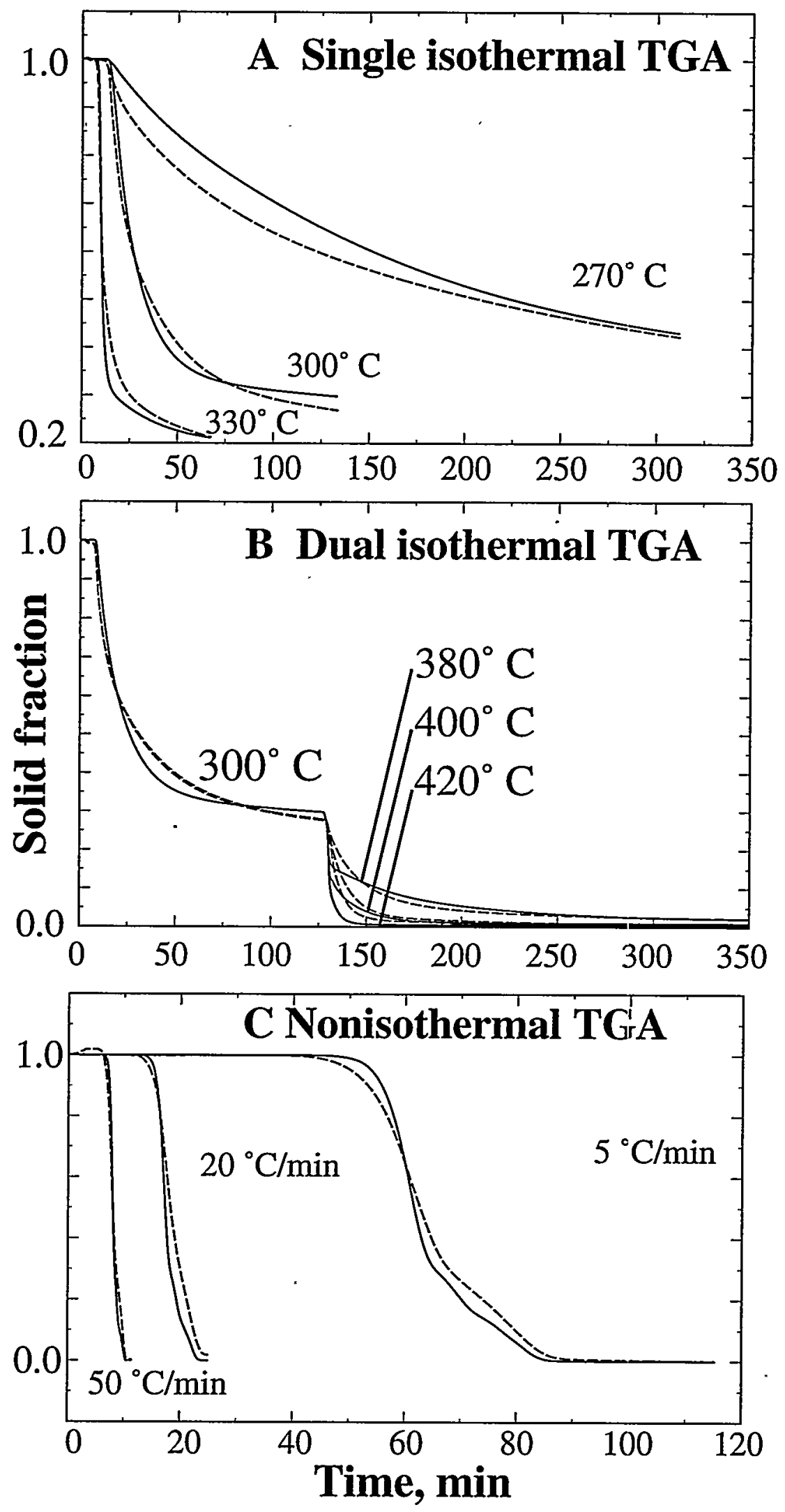

Fig. 9 


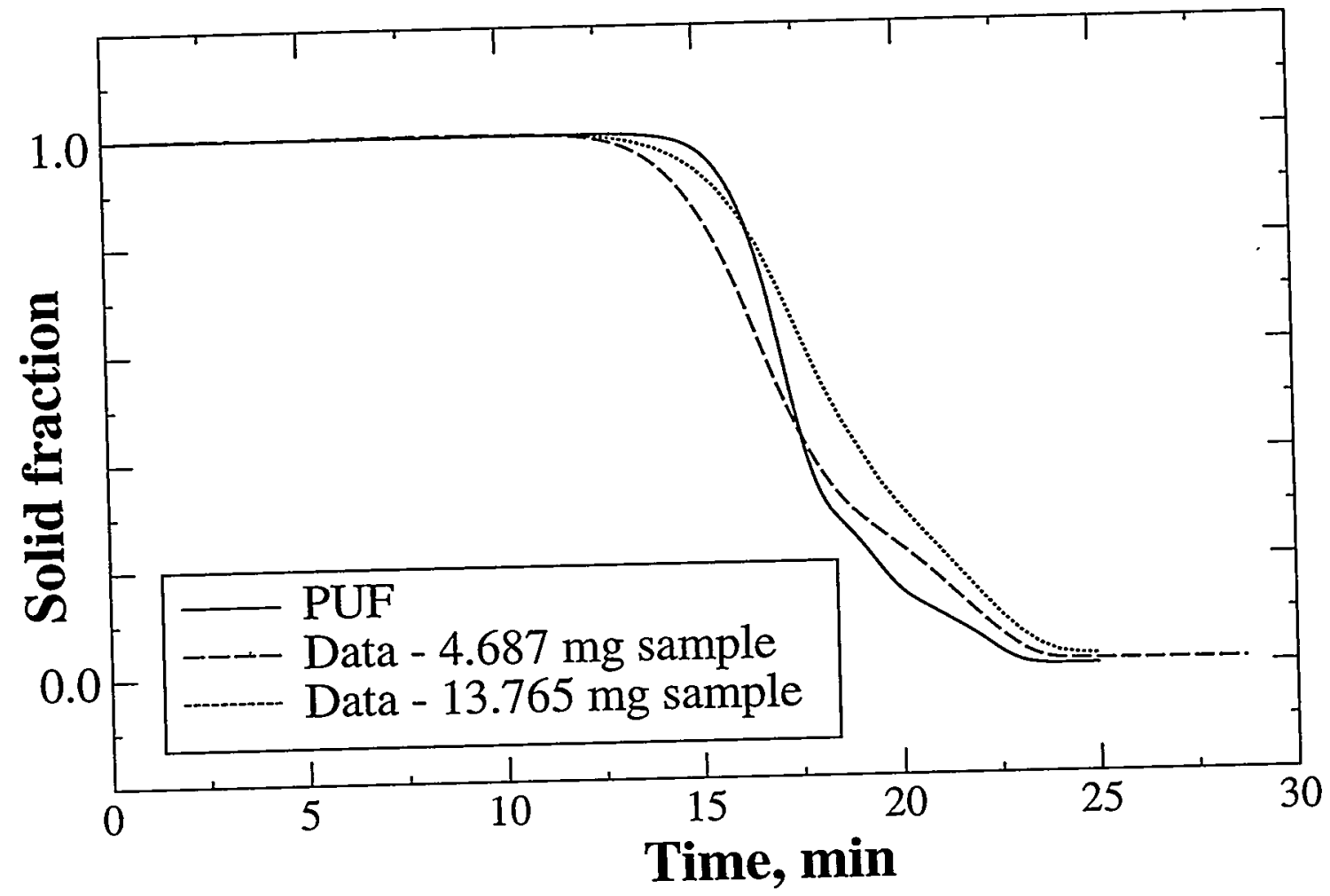

Fig. 10 


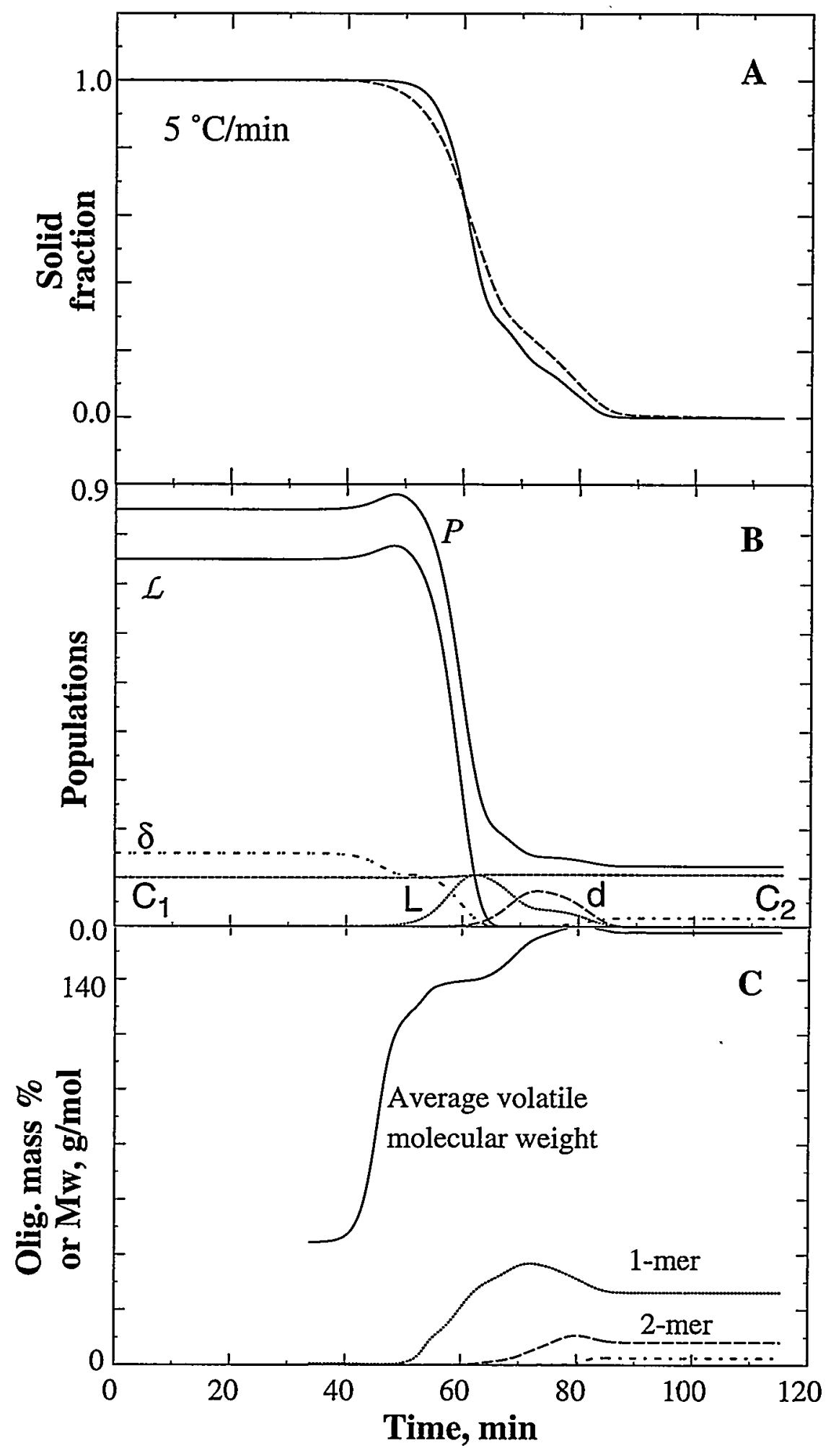

Fig. 11 


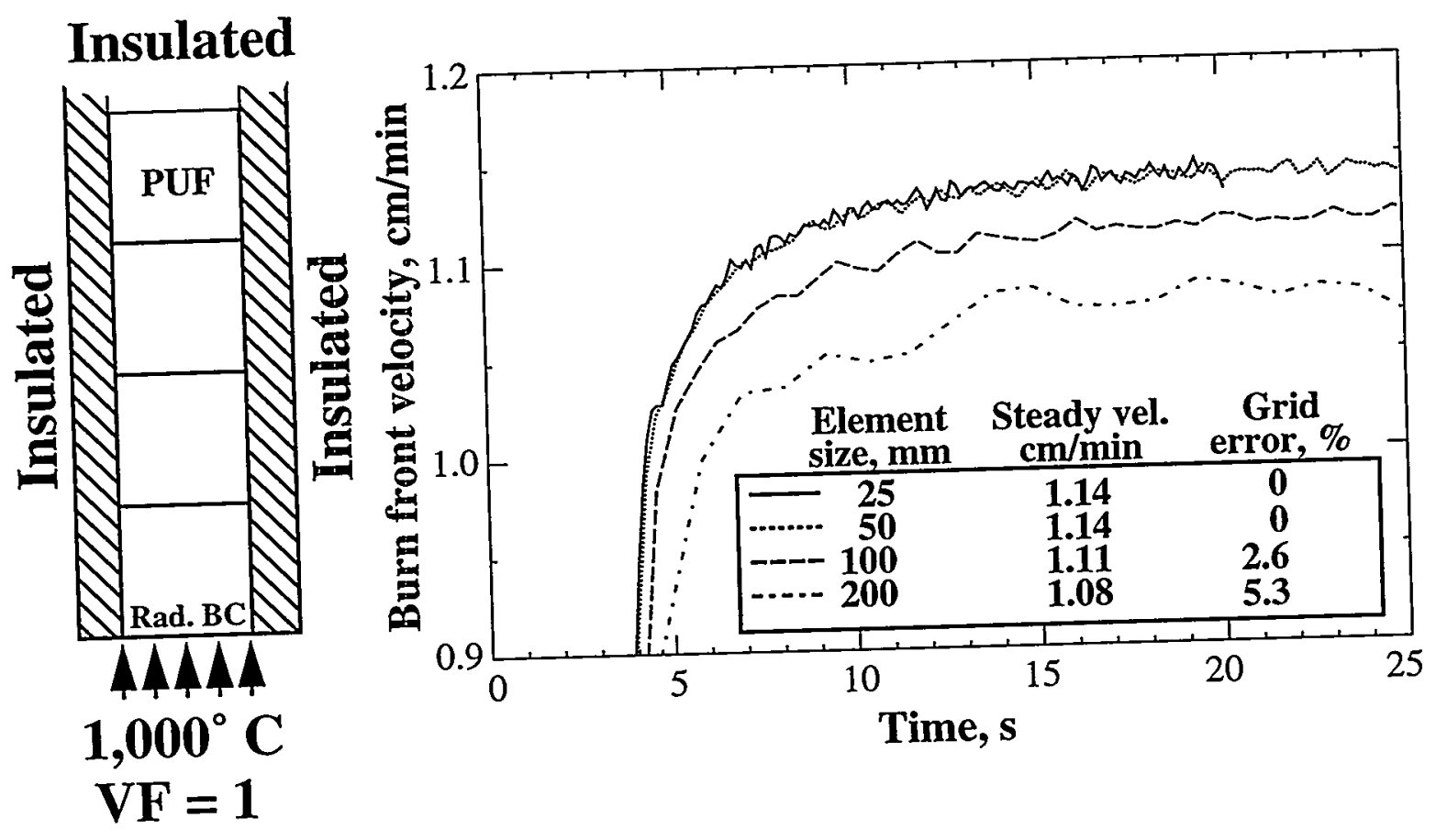

Fig. 12 


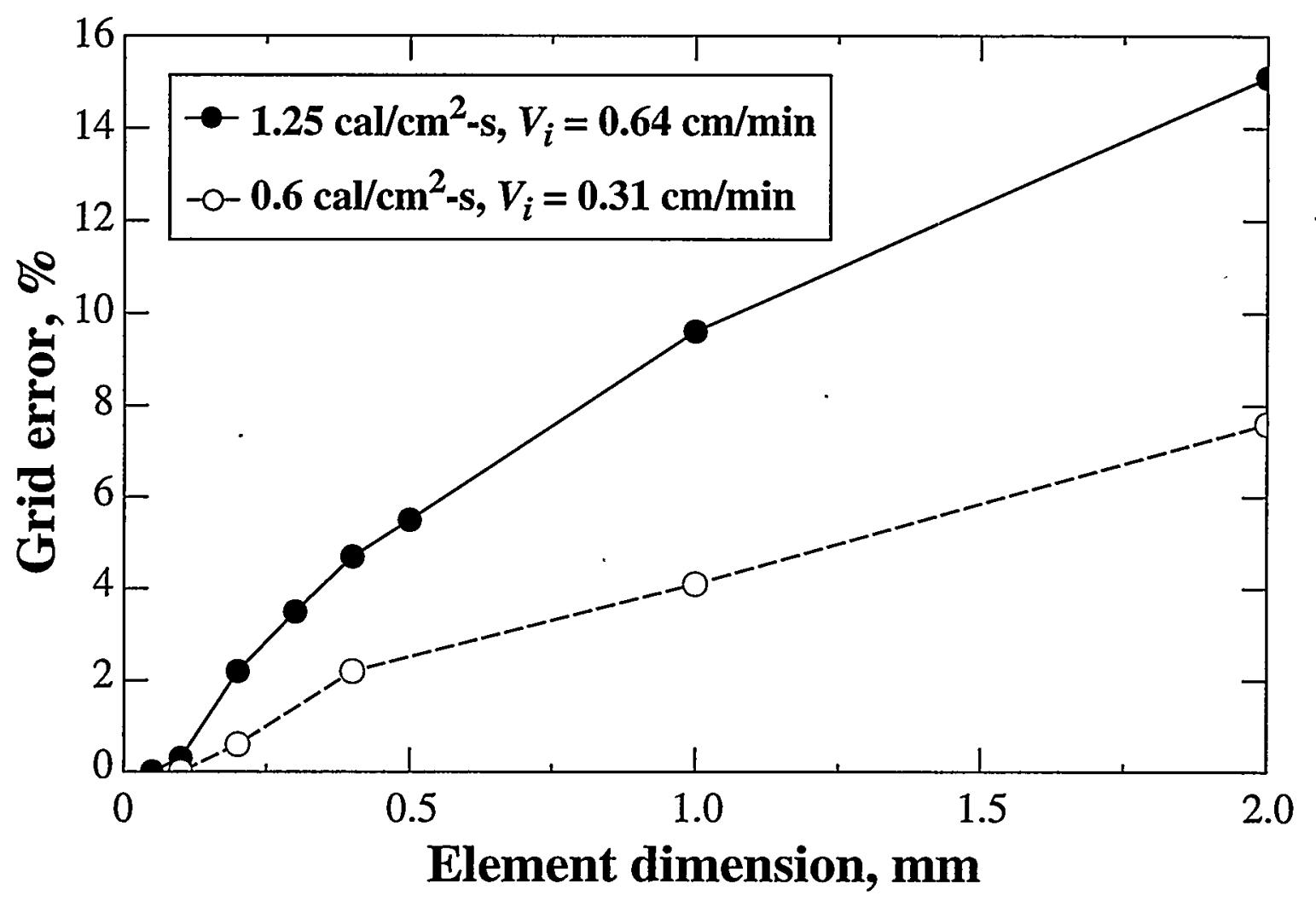

Fig. 13 
Heat lamp array
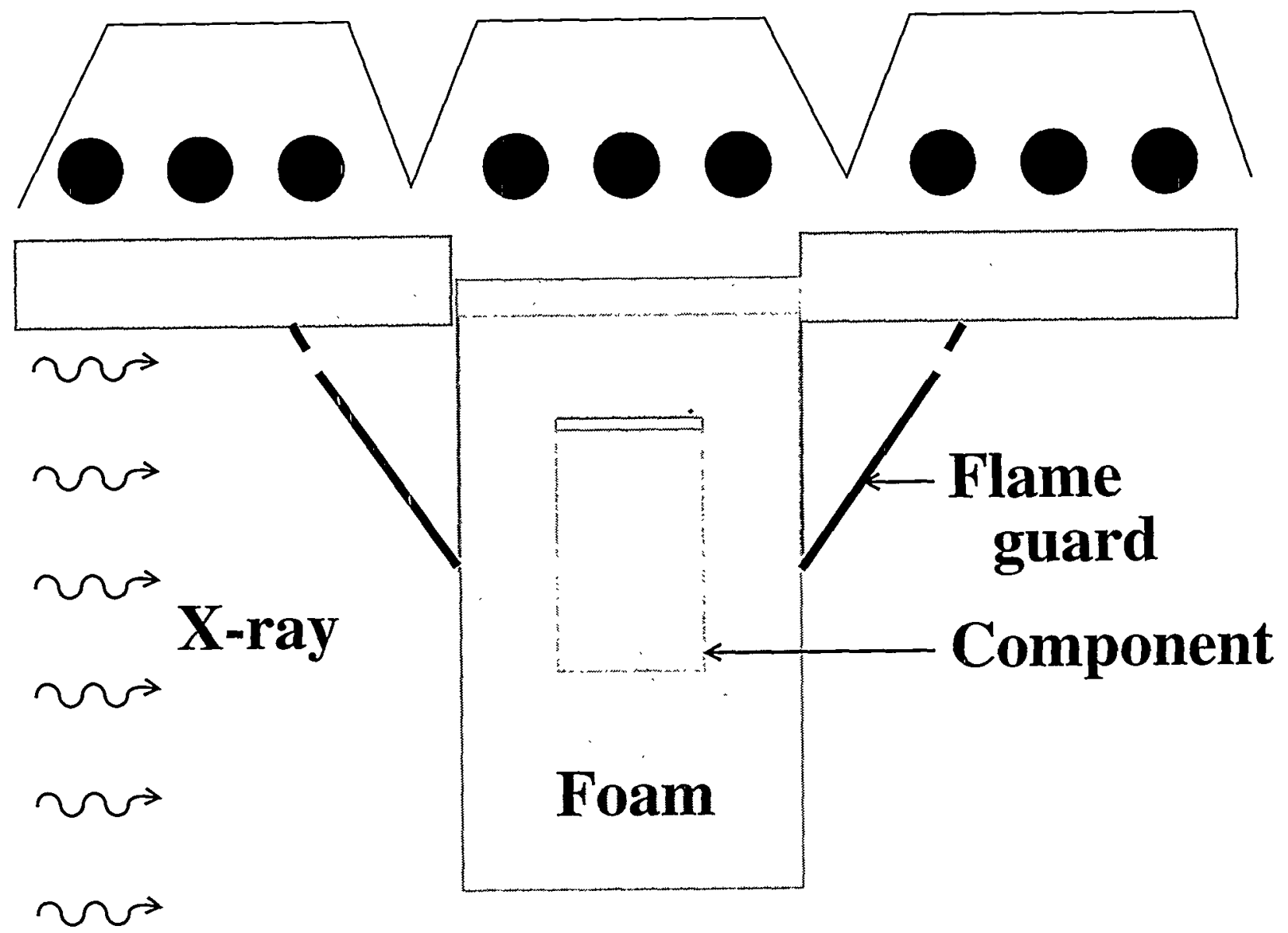

Fig. 14 
Slot in tube for bottom plate thermocouple

303 SS bottom plate press fit into tube

$6 \mathrm{~mm}$ diameter weep holes

Cup thermocouples

$321 \mathrm{SS}$ tube $(0.5 \mathrm{~mm}$ wall)

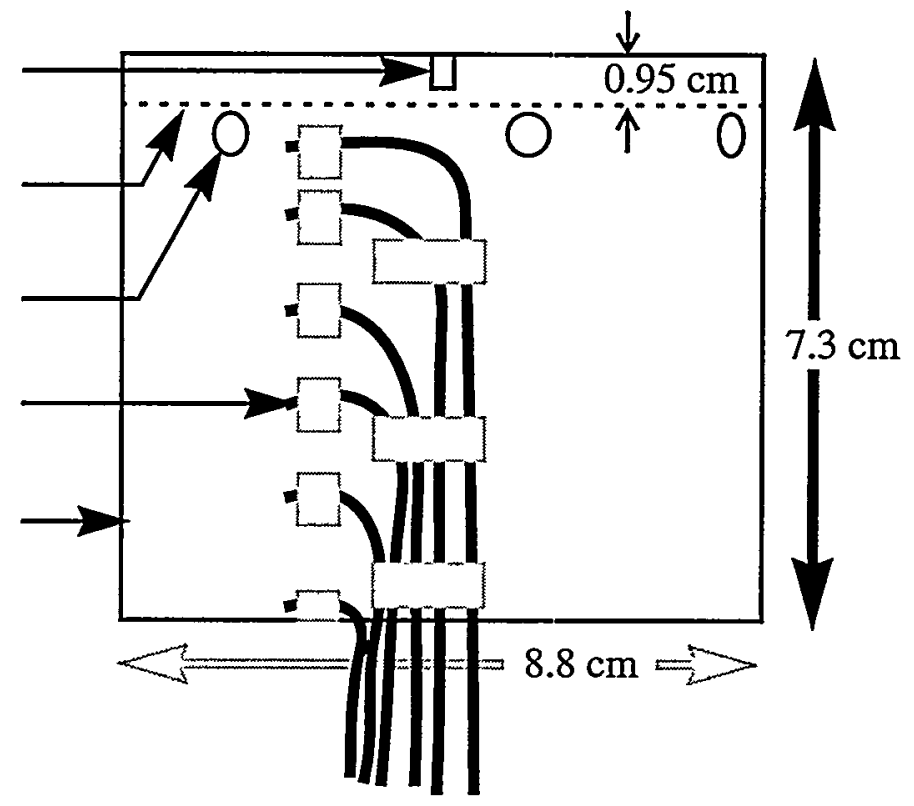

Fig. 15 


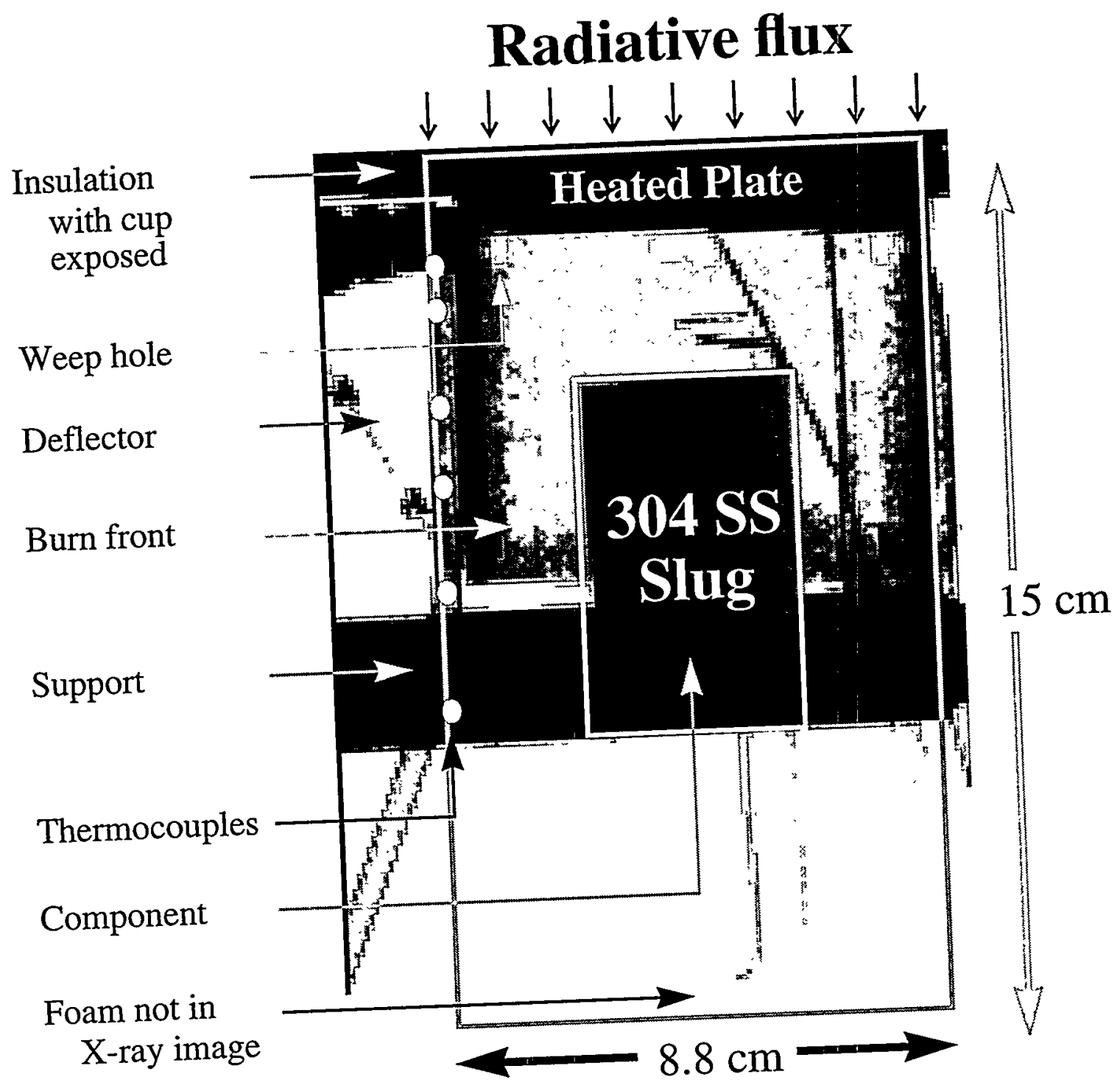

Fig. 16 


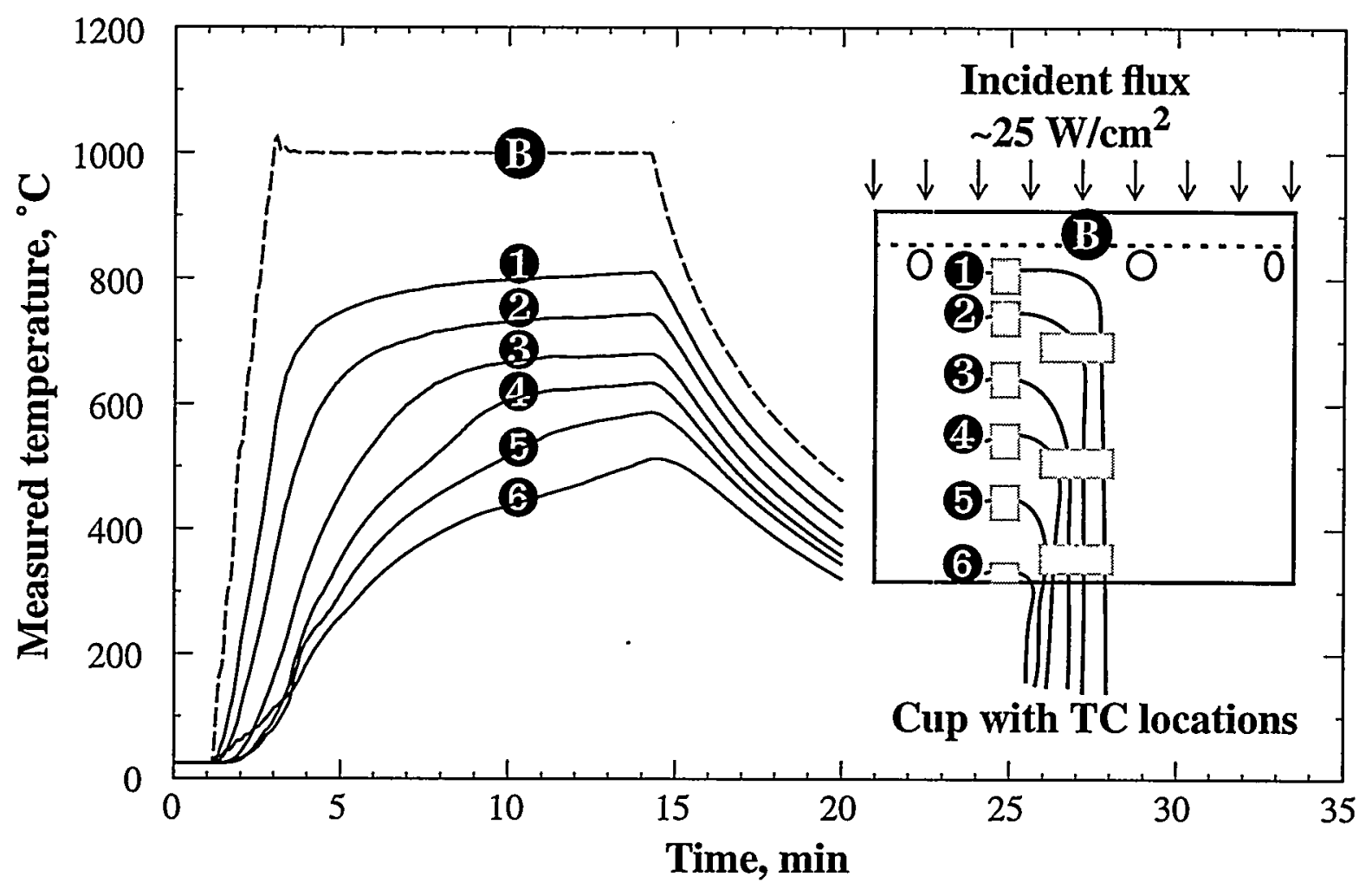

Fig. 17 


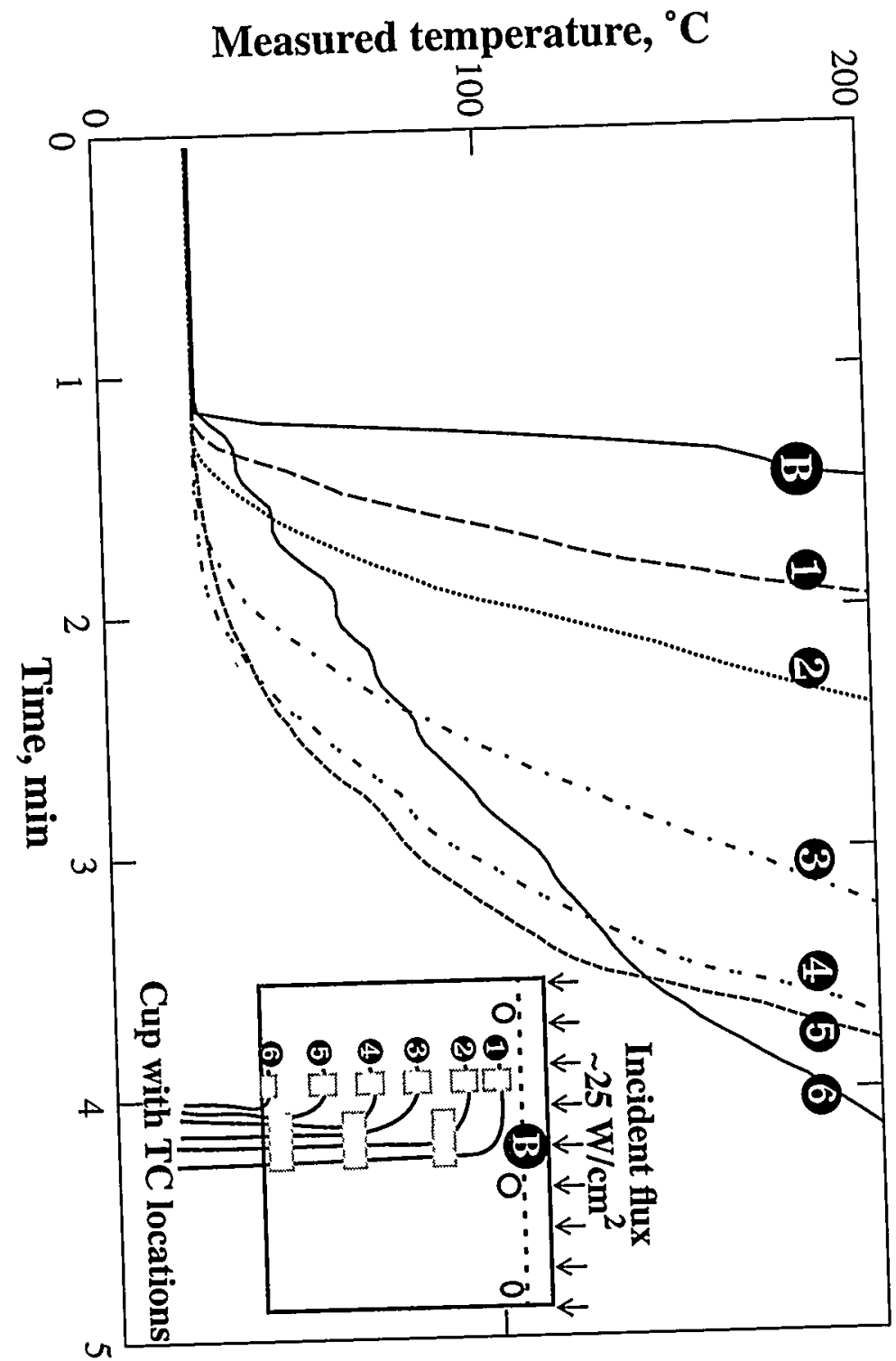




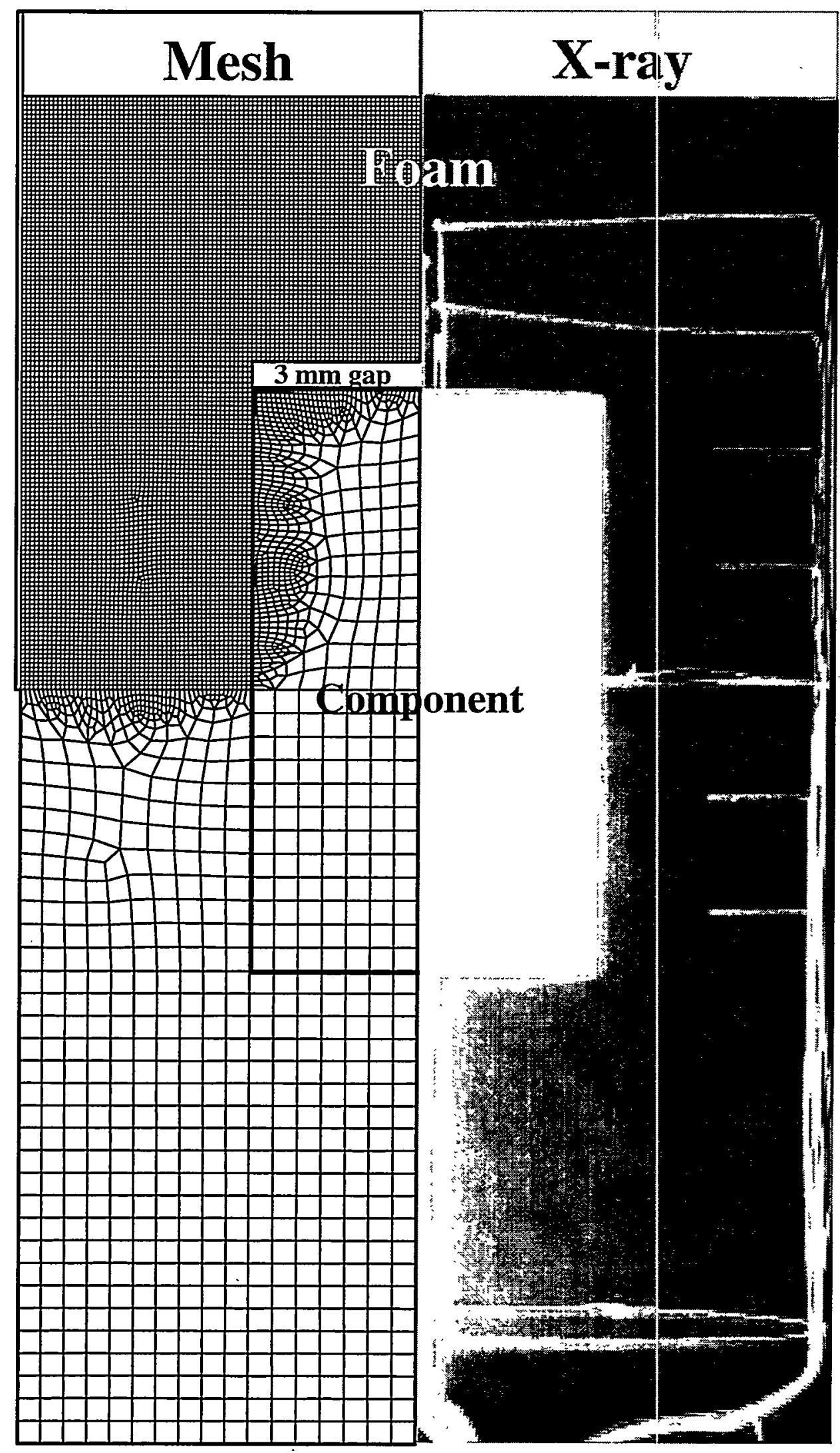

Fig. 19 


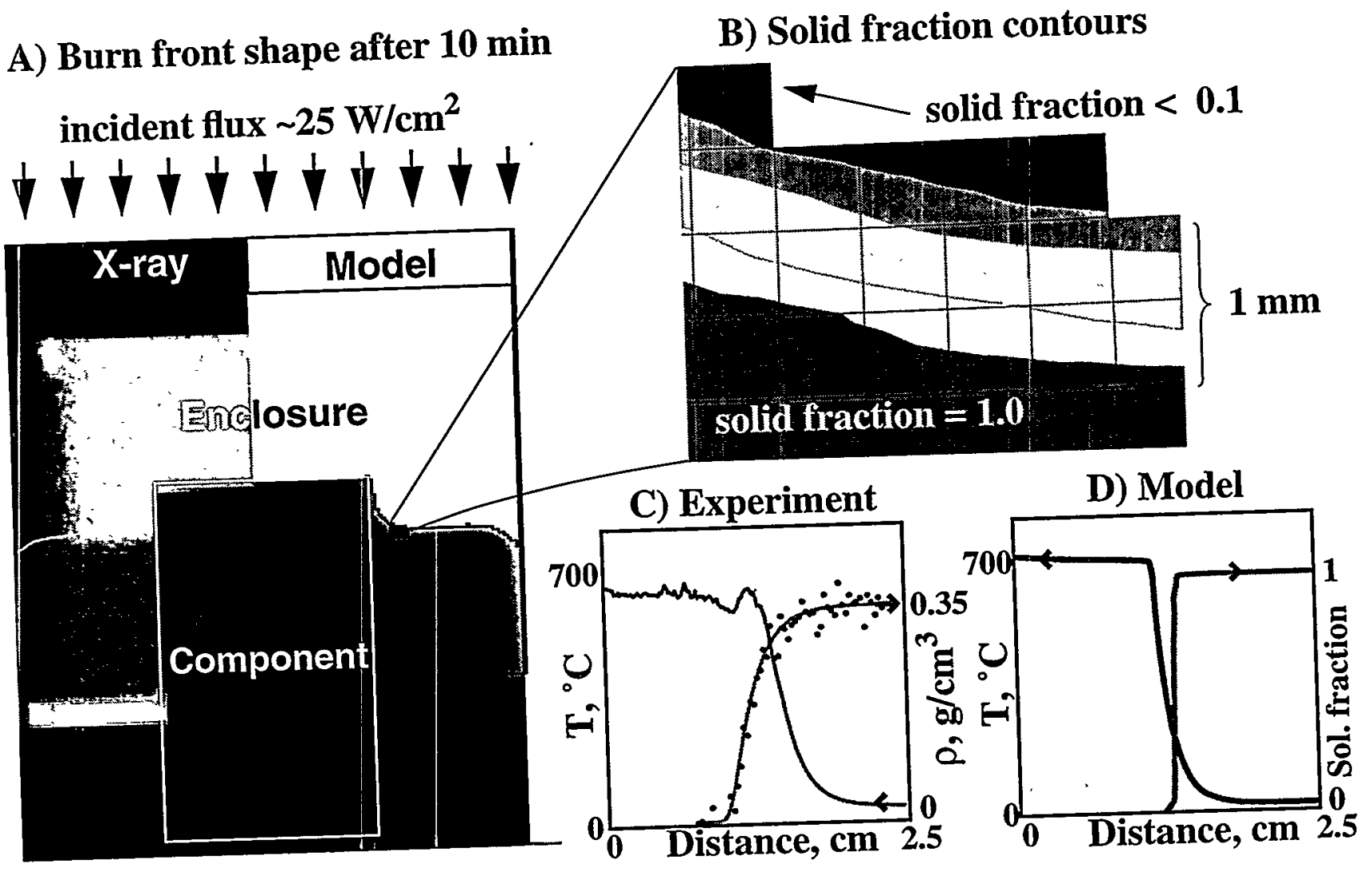

Fig. 20 

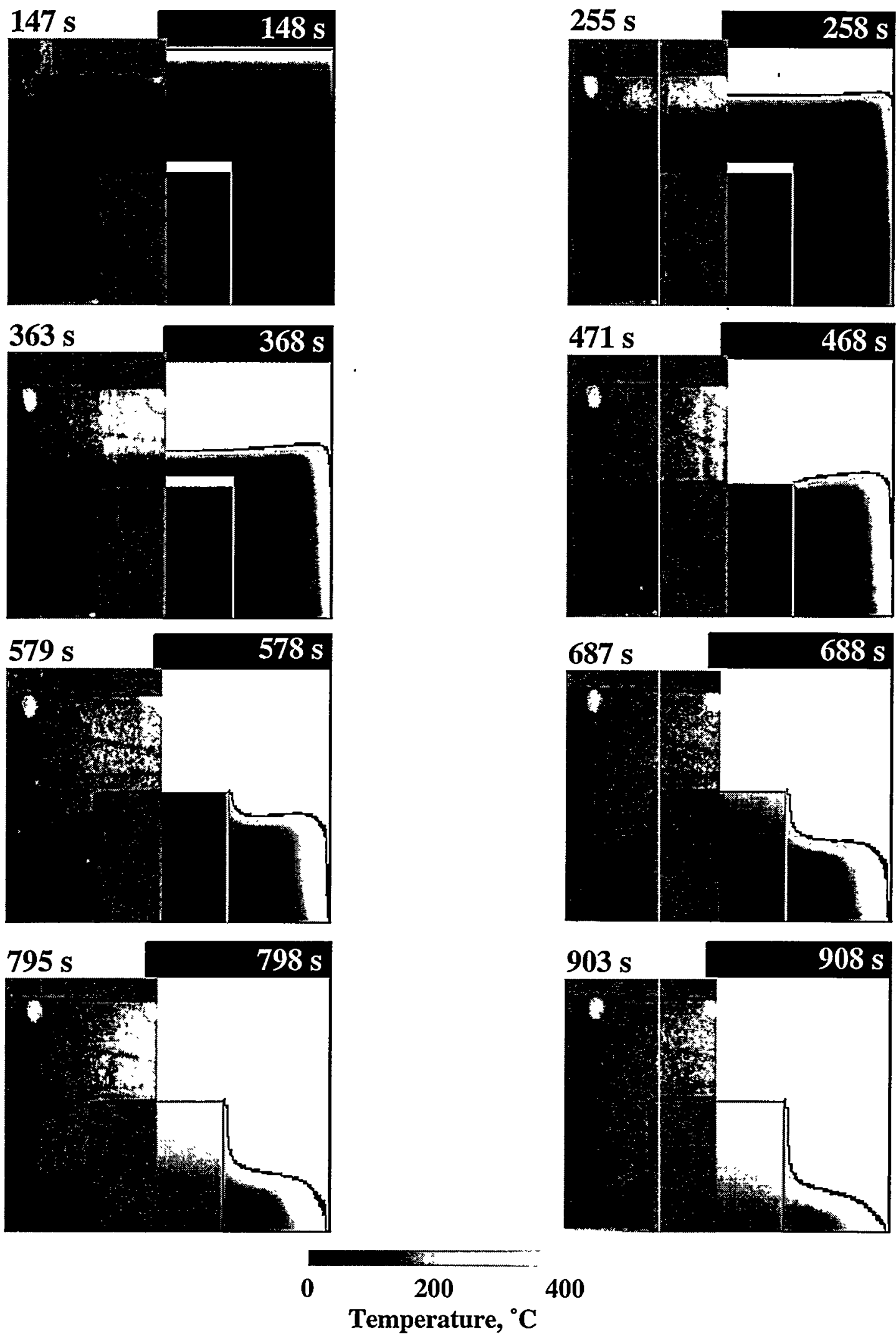

Fig. 21 
$\sigma$

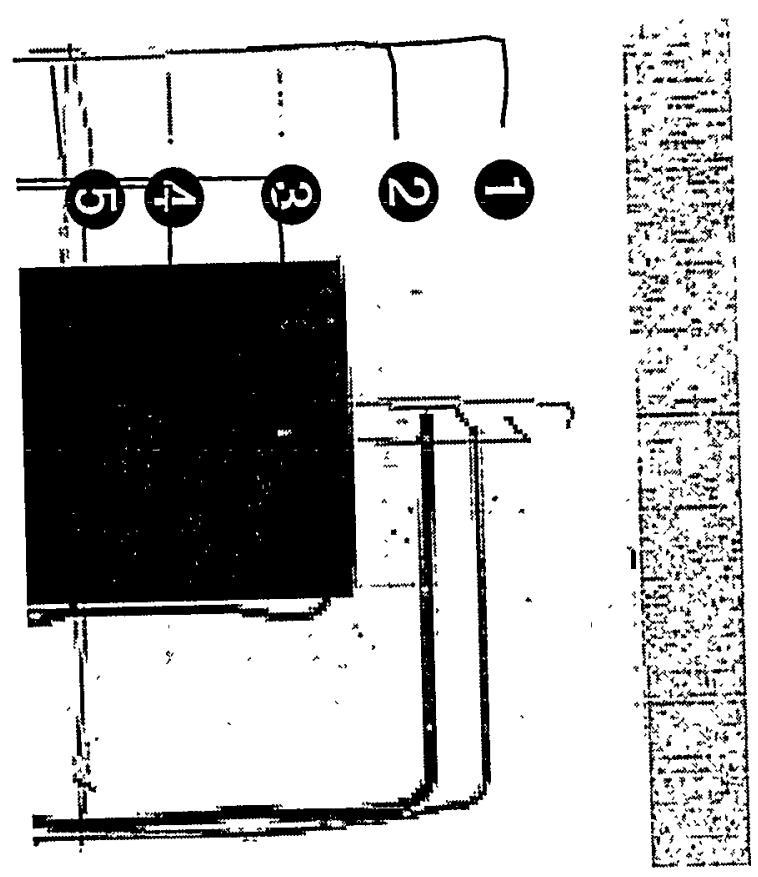

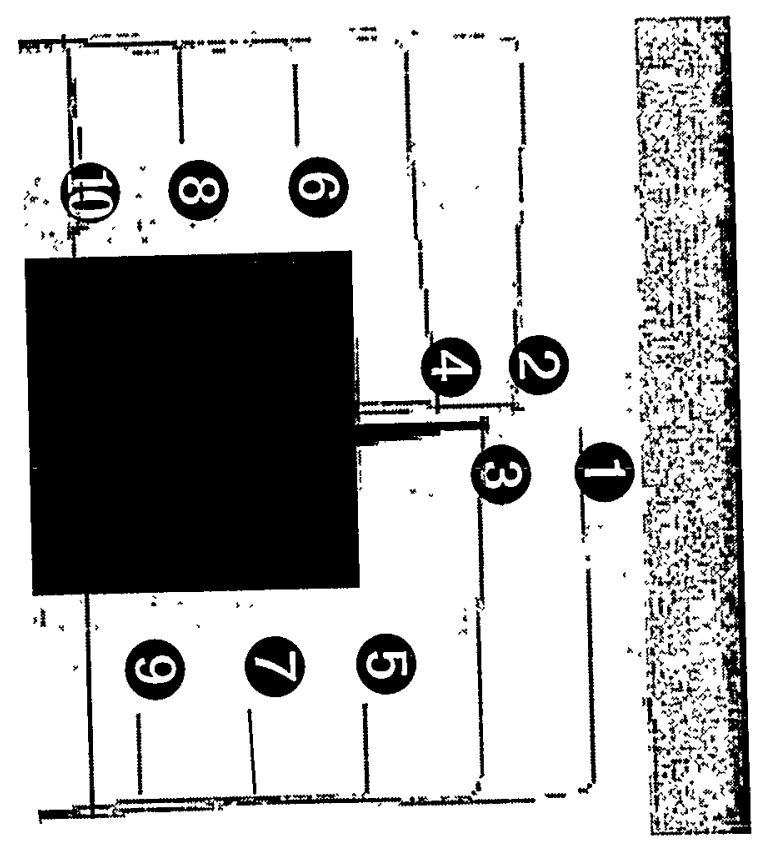



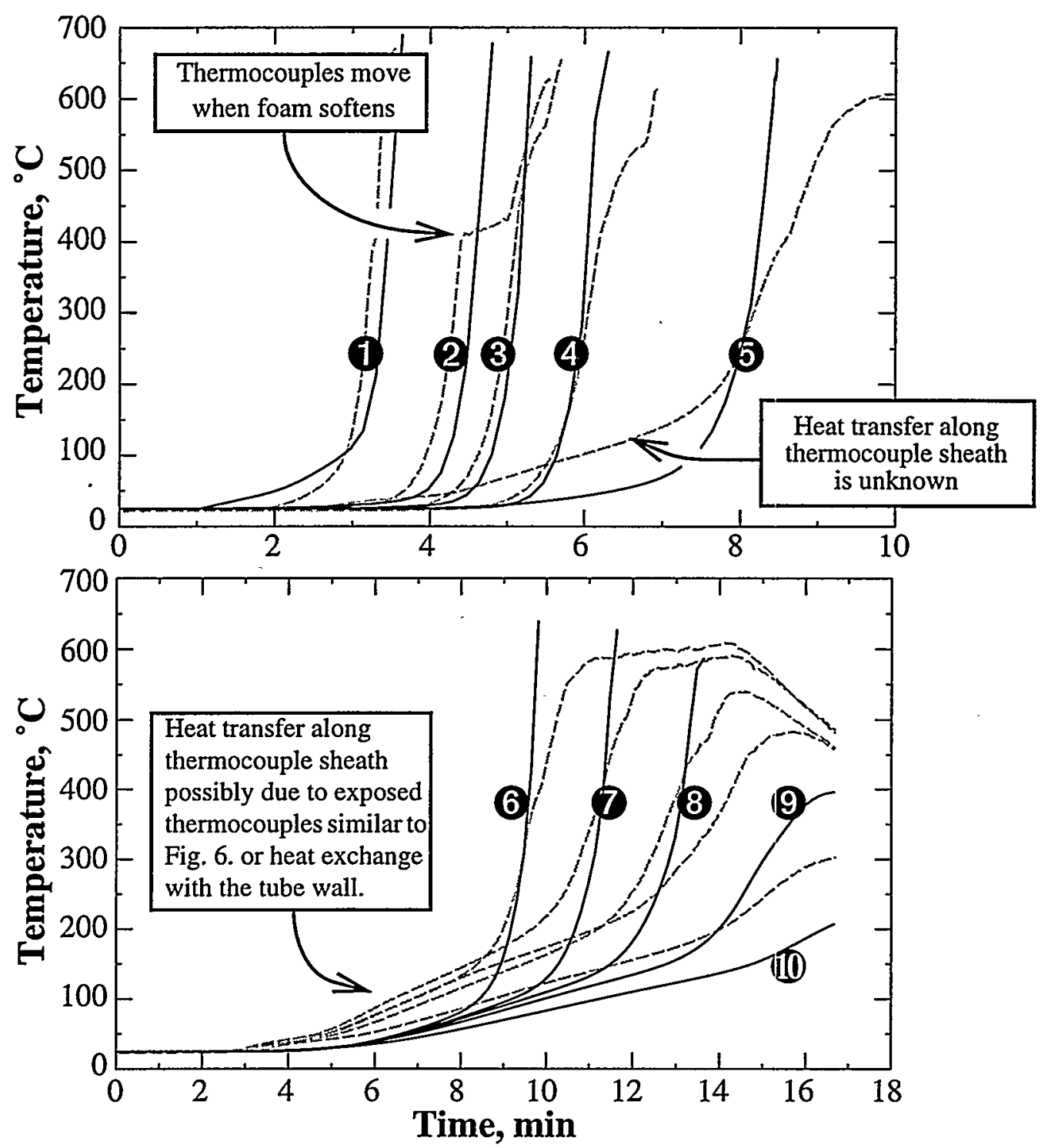

Fig. 23 


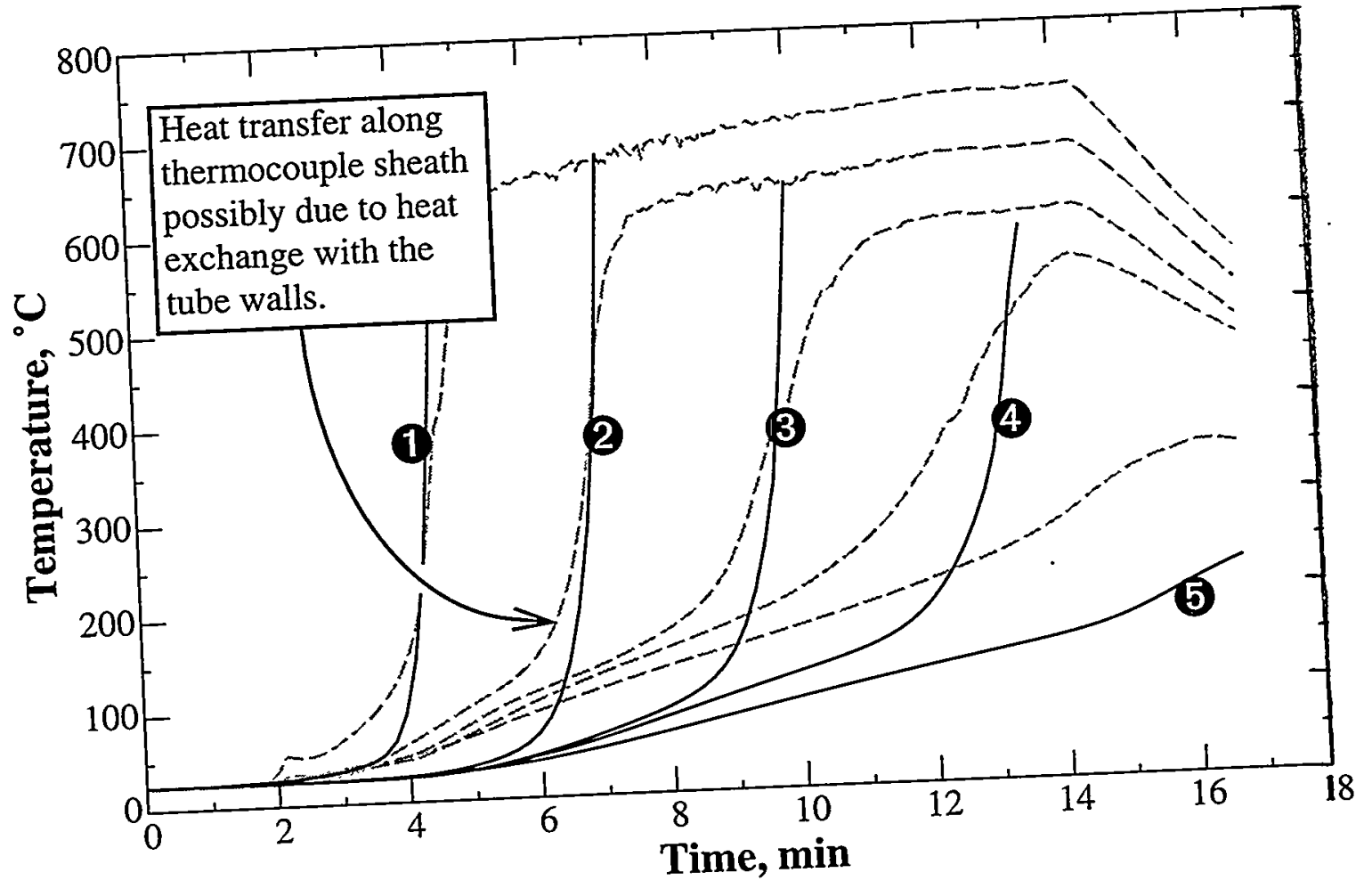

Fig. 24 


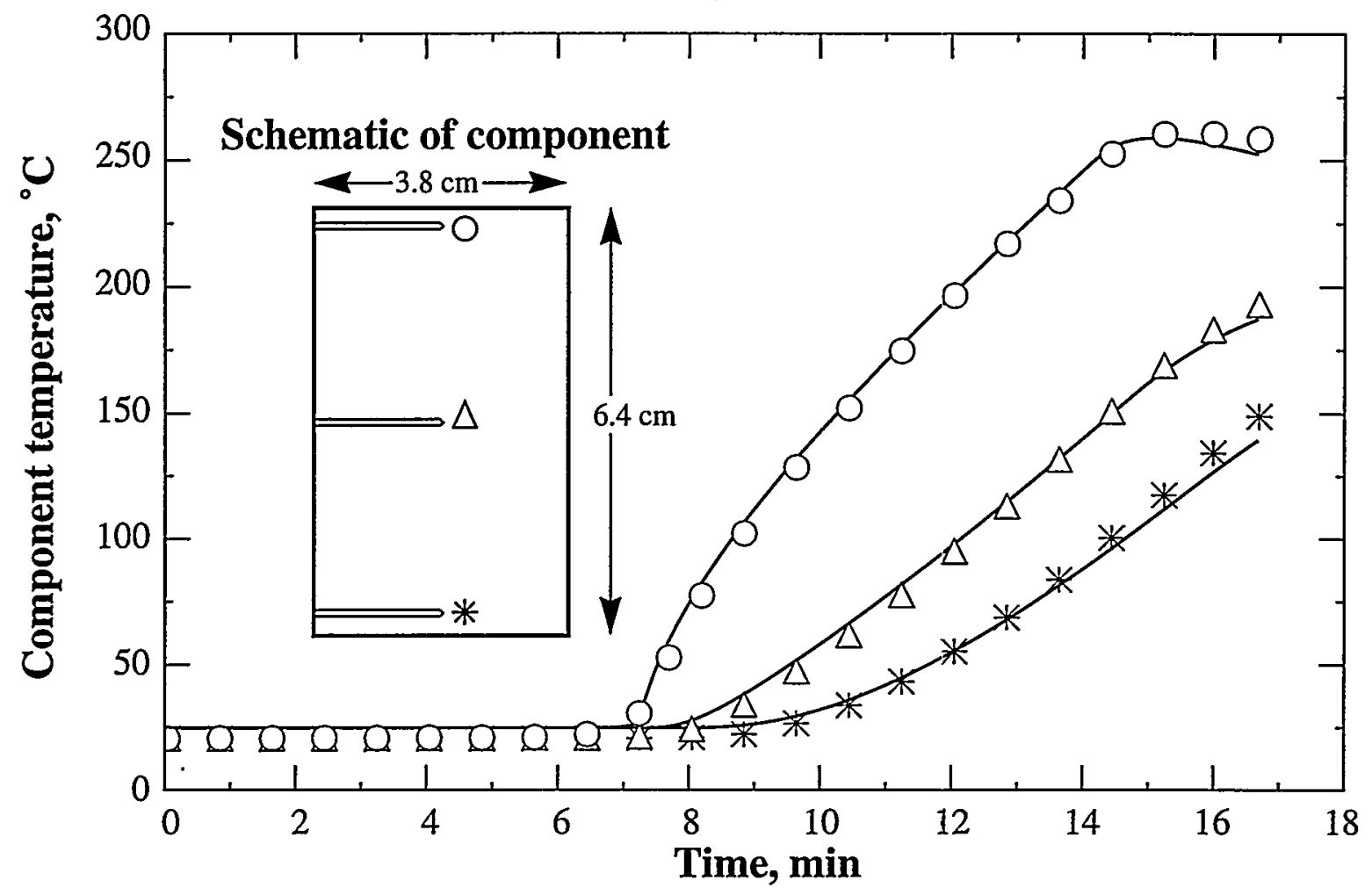

Fig. 25 
A) Top view of exposed thermocouple

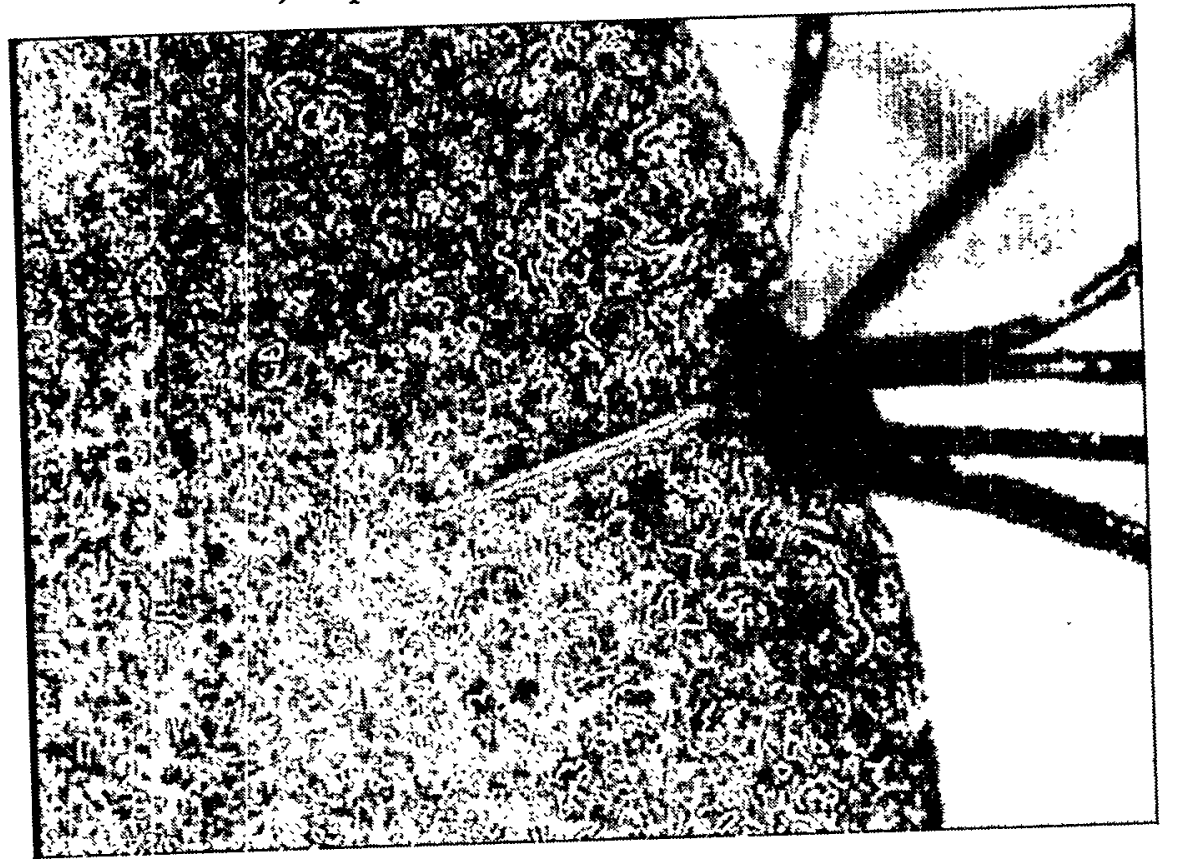

B) Side view of exposed thermocouple

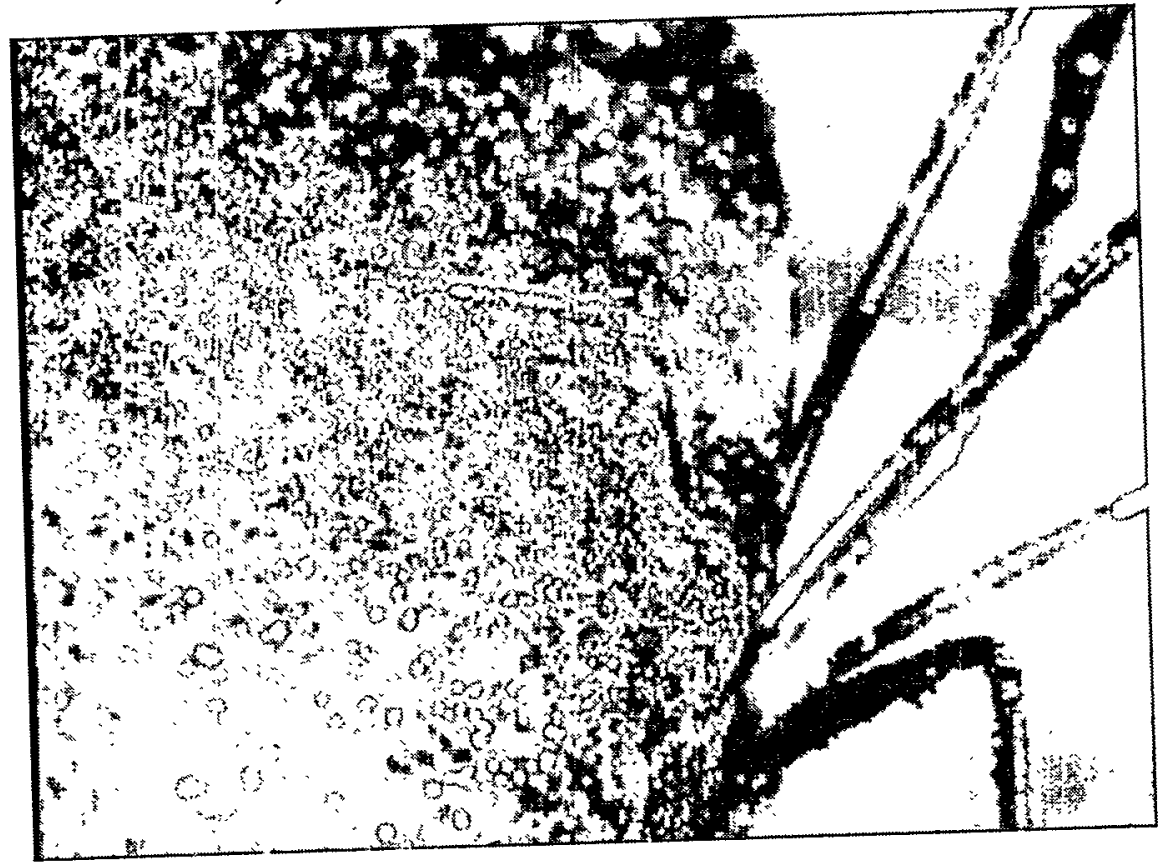

Fig. 26 


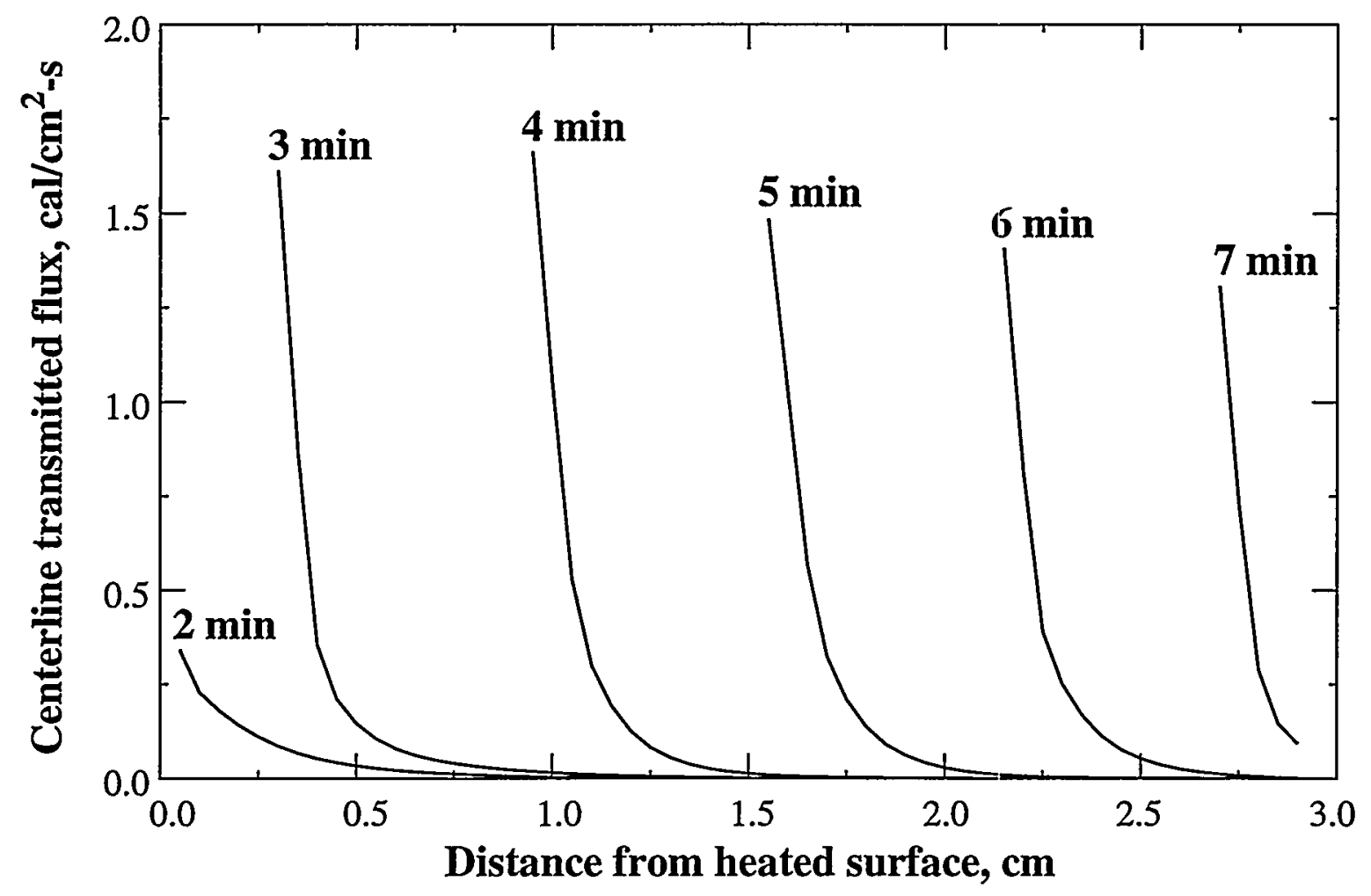

Fig. 27 


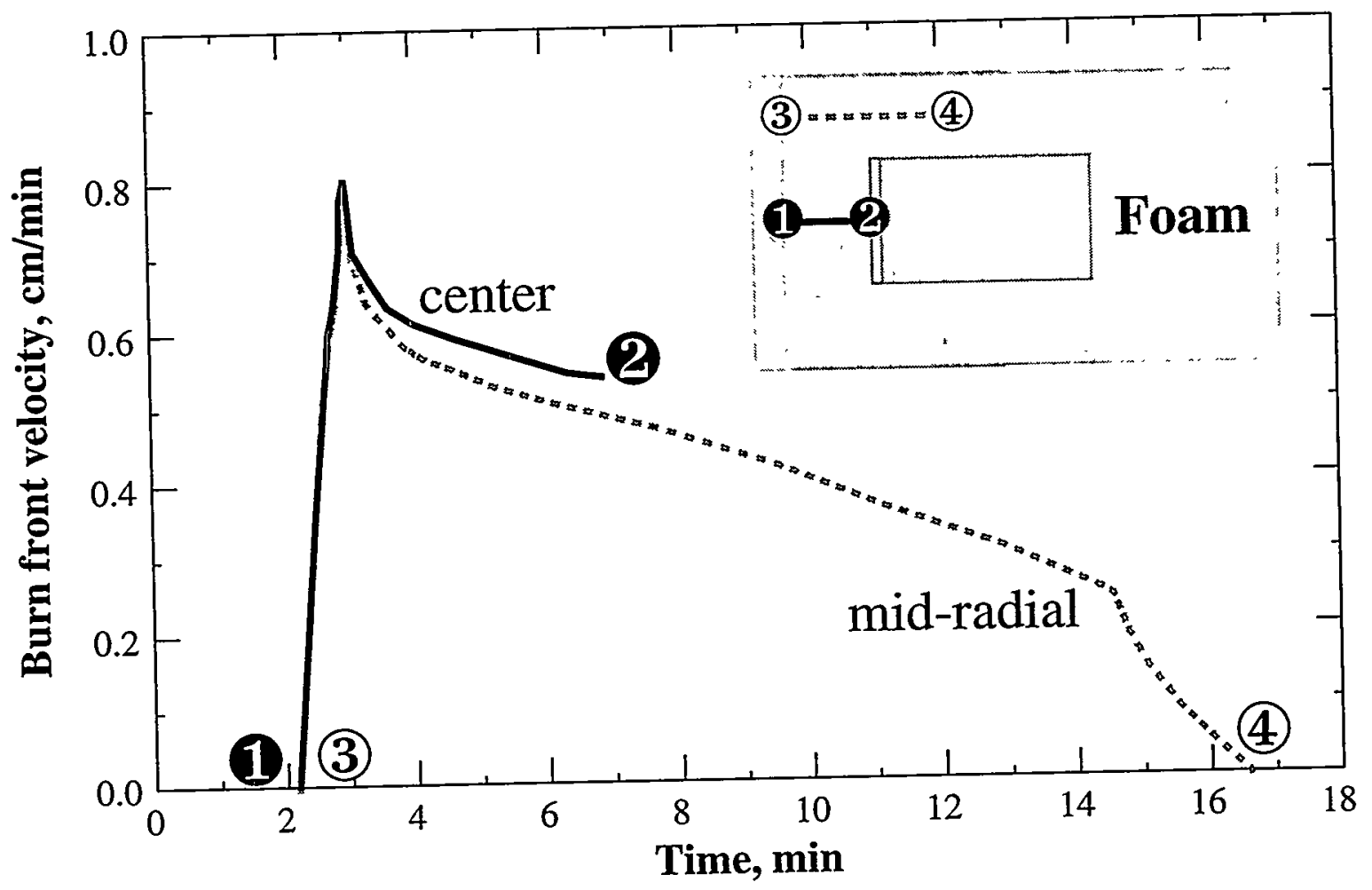

Fig. 28 